\title{
The effect of free-surface tension on scattering of water waves by small bottom undulation
}

\author{
Smrutiranjan Mohapatra ${ }^{1}$
}

(Received 22 August 2016; revised 16 February 2017)

\begin{abstract}
We examine the influence of surface tension at the free-surface on the problem of scattering of normal incident waves by small bottom undulation in a two-layer fluid within the framework of linearised wave theory. In such a two-layer fluid, there exist two different modes of time-harmonic waves for the given frequency, one of which corresponds to a free-surface disturbance and the other to an interfacial wave motion. Using perturbation technique, the problem is reduced up to first-order to a coupled boundary value problem which is solved by a method based on Green's integral theorem with the introduction of appropriate Green's functions. The first-order approximations to the reflection and transmission coefficients evaluated in terms of computable integrals for both the modes. For a sinusoidal bottom topography, these coefficients are determined and the numerical results depicted graphically. When
\end{abstract}

DOI:10.21914/anziamj.v58i0.11232, (c) Austral. Mathematical Soc. 2017. Published March 9, 2017. ISSN 1445-8810. (Print two pages per sheet of paper.) Copies of this article must not be made otherwise available on the internet; instead link directly to the DOI for this article. Record comments on this article via http://journal . austms . org . au/ojs/index.php/ANZIAMJ/comment/add/11232/0 
the wave is incident due to the free-surface disturbance or the interfacial wave motion in a two-layer fluid, we always find energy transfer from the incident wave mode to the other wave mode. We conclude that realistic changes in surface tension on the free-surface have a significant effect on the problem of water wave scattering by bottom undulation in a two-layer fluid.

Subject class: primary 76B15; secondary 76B55

Keywords: two-layer fluid; surface tension; bottom undulation; Green's function; perturbation technique; reflection coefficient; transmission coefficient.

\section{Contents}

1 Introduction

2 Mathematical formulation

E42

3 Method of solution

E47

3.1 Perturbation technique . . . . . . . . . . . E48

3.2 Introduction of Green's functions . . . . . . . . . E50

4 Reflection and transmission coefficients

E55

5 Special form of the bottom topography

E57

6 Numerical results

E59

7 Conclusion

E75

References 


\section{Introduction}

Scattering of water waves by an obstacle or a geometrical disturbance at the bottom of an ocean creates interesting mathematical problems drawing attention of various types by the marine and ocean researchers for obtaining their useful solution (Chapman and Porter [2], Davies [3], Mei [13], Miles [14], and Porter and Porter [19]). The study of scattering of waves in a two-layer fluid has drawn reasonable attention due to various applications in the areas of coastal and marine engineering, and such problems have been investigated for a long time (Chamberlain and Porter [1], Linton and McIver [9], Maiti and Mandal [11], and Mohapatra and Bora [15, 16, 17]). The problem of reflection of water waves by a patch of bottom undulation is important in the development of bottom-parallel bars or pipes (Mandal and De [12]).

Lamb [8] describes the linearized theory of small amplitude waves in two superposed inviscid fluids, separated by a common interface, and the upper layer fluid of lower density having a free-surface. In such a two-layer fluid region, for a given frequency, time-harmonic gravity waves of two different modes propagate in the positive $x$-direction. When a train of waves of a particular mode encounters an obstacle, then some of the energy from the incident wave mode is transferred to the other mode due to scattering by the obstacle. If the bed of the ocean has an undulation, then the wave train is partially reflected by it, and partially transmitted over it. However, there exists a class of mostly naturally occurring bottom standing obstacles for which we apply perturbation technique for obtaining first-order corrections to the reflection and transmission coefficients (Mohapatra and Bora[18]).

Very few attempts have been made to include the effect of surface tension in water wave problems in a single-layer fluid involving a fixed or floating structure (Evans [4, 5], Harter et al. [6, 7] and Rhodes-Robinson [20, 21]). An ocean contains more than one fluid layer (e.g., fresh water layer, salt water layer, mud layer) and the scattering of waves in a such fluid is quite important. This motivated us to study the scattering problems in a two-layer fluid in the 
presence of surface tension at the free-surface. The inclusion of surface tension in the linear water-wave problem introduces an additional term in the freesurface boundary condition (Rhodes-Robinson [20]). In section 2, the problem is formulated for scattering of water waves by small bottom undulation. We consider a two-layer fluid whose upper layer is bounded above by a free-surface and the lower layer encounters a bottom undulation. The importance of the present study is that the effect of surface tension at the free-surface of a two-layer fluid is included. No work has till now been carried out in two-layer fluid scattering problems where surface tension has been included in either of the layers. In this case, time-harmonic waves of a particular frequency propagate with two different wavenumbers: one of which corresponds to a free-surface disturbance on the fluid; and the other to an interfacial wave motion in a two-layer fluid (Linton and Cadby [10]). Applying perturbation techniques in Section 3, we reduce the original problem to a simpler one for the first-order corrections of the potentials. The solution of this boundary value problem is then obtained by an appropriate use of Green's integral theorem to the potential functions describing the problem. In Section 4, the reflection and transmission coefficients are evaluated approximately up to the first-order in terms of integrals involving the shape function of bottom undulation. Sections 5 and 6 present a special form of bottom undulation, that is, a patch of sinusoidal ripples and the first-order coefficients are depicted graphically for various values of different parameters. The paper concludes with a brief discussion in Section 7 .

\section{Mathematical formulation}

We consider an inviscid and incompressible two-layer fluid of which the upper layer is of finite height and has a free surface, and the lower layer has an undulating bottom topography. We further assume that the fluid motion is irrotational and simple harmonic in time with frequency $\omega$. Each fluid is of infinite horizontal extent in the $\boldsymbol{x}$-direction while the depth is along $\mathbf{y}$-direction 
Figure 1: Domain definition sketch.

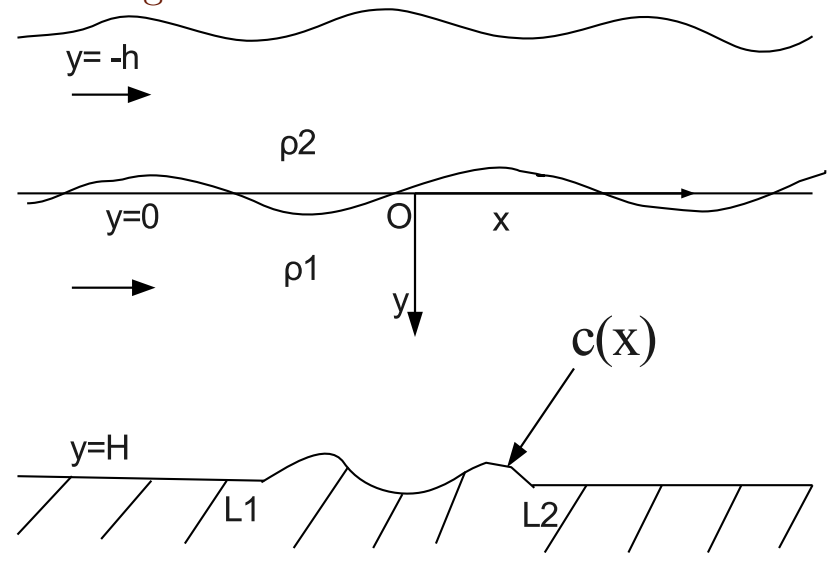

which is considered vertically downwards with $y=-h$ as the mean position of the free-surface (Figure 1). The origin $\mathrm{O}$ is at the undisturbed interface between the upper and lower fluids, and $y=0$ as the mean position of the interface of the layers. The lower layer fluid is of uniform finite depth $\mathrm{H}$ far to either side of the undulation. Here, the bottom with small undulation is described by $y=H+\epsilon c(x)$, where $c(x)$ is a bounded and continuous function describing the shape of the undulation and $c(x) \rightarrow 0$ as $|x| \rightarrow \infty$, and the non-dimensional number $\epsilon \ll 1$ is a measure of smallness of the undulation. Let $\phi(x, y)$ and $\varphi(x, y)$, respectively, be complex-valued potential functions for the lower layer fluid $(0<y<H)$ of density $\rho_{1}$ and the upper layer fluid $(-h<y<0)$ of density $\rho_{2}\left(<\rho_{1}\right)$. Under the usual assumptions of linear water wave theory, define the time dependent velocity potentials in the lower and upper layer, respectively, in the form $\mathfrak{R}\left[\phi(x, y) e^{-i \omega t}\right]$ and $\mathfrak{R}\left[\varphi(x, y) e^{-i \omega t}\right]$.

The governing equation for the coupled boundary value problem involving these potentials $\phi$ and $\varphi$ is Laplace's equation:

$$
\begin{aligned}
& \nabla^{2} \phi=0 \quad \text { in the lower fluid, } \\
& \nabla^{2} \varphi=0 \quad \text { in the upper fluid. }
\end{aligned}
$$


Define $\rho=\rho_{2} / \rho_{1}$ and $K=\omega^{2} / g ; g$ is the acceleration due to gravity; $S=\tau /\left(\rho_{2} g\right)$ where $\tau$ is the coefficient of surface tension; and $\frac{\partial}{\partial n}$ the derivative normal to the bottom at a point $(x, y)$. The linearized boundary conditions at the bottom surface, on the interface, and at the free-surface are

$$
\begin{aligned}
\frac{\partial \phi}{\partial \mathfrak{n}} & =0 \quad \text { on } \mathrm{y}=\mathrm{H}+\epsilon \mathrm{c}(\mathrm{x}), \\
\frac{\partial \phi}{\partial y} & =\frac{\partial \varphi}{\partial y} \quad \text { on } y=0 \\
\mathrm{~K} \phi+\frac{\partial \phi}{\partial y} & =\rho\left(\mathrm{K} \varphi+\frac{\partial \varphi}{\partial y}\right) \quad \text { on } y=0 \\
\mathrm{~K} \varphi+\frac{\partial \varphi}{\partial y}+\mathrm{S} \frac{\partial^{3} \varphi}{\partial y^{3}} & =0 \quad \text { on } y=-\mathrm{h} .
\end{aligned}
$$

Equation (6) includes surface tension in the free-surface condition. This condition is derived from Bernoulli's equation and the surface tension condition of discontinuous pressure across the free-surface (Evans [5]).

Within this framework in a two-layer fluid, a train of progressive waves takes the form (up to an arbitrary multiplicative constant)

$$
\begin{aligned}
\phi & =e^{ \pm i u x} \cosh [u(H-y)] \quad \text { in } 0 \leqslant y \leqslant H \\
\varphi & =e^{ \pm i u x} Z(u, y) \quad \text { in }-h \leqslant y \leqslant 0 \\
Z(u, y) & =\frac{\sinh u H\left\{\left(1+S u^{2}\right) u \cosh [u(h+y)]-K \sinh [u(h+y)]\right\}}{K \cosh u h-\left(1+S u^{2}\right) u \sinh u h},
\end{aligned}
$$

and $u$ satisfying the dispersion relation $\Delta(\mathfrak{u})=0$, where

$$
\begin{aligned}
\Delta(u)= & K^{2} \cosh u h \cosh u H+\left[u^{2}(1-\rho)\left(1+S u^{2}\right)+K^{2} \rho\right] \sinh u h \sinh u H \\
& -K u\left[\left(1+S u^{2}\right) \sinh u h \cosh u H+\left(1+\rho S u^{2}\right) \sinh u H \cosh u h\right] .
\end{aligned}
$$

We obtained the dispersion equation (10) when solving equations (1) and (2), by using the boundary conditions (4-6) and $\partial \phi / \partial y=0$ on $y=H$. If we take $S=0$ (i.e., the surface tension is absent on the free-surface), then the above 
dispersion equation (10) coincides with the corresponding dispersion equation obtained previously in those cases where the two-layer fluid had a free-surface and a bottom undulation (Maiti and Mandal [11]). In the dispersion equation, there are two positive real roots $p_{1}$ and $p_{2}$ that indicate the propagating modes and a countable infinity of purely imaginary roots $i \kappa_{n}(n=1,2, \ldots)$ that characterise a set of evanescent modes. Since equation (10) has exactly two non-zero positive real roots $p_{1}$ and $p_{2}\left(p_{1}<p_{2}\right.$, say), so there exist two modes of waves, one of which corresponds to a free-surface disturbance and the other to an interfacial wave motion propagating along the positive $x$-direction (Linton and Cadby [10]). However, there exists only one mode of waves propagating on the interface along the positive $x$-direction in the case of a two-layer fluid flowing over a bottom undulation, where the upper layer fluid is bounded above by a rigid horizontal surface (Mohapatra and Bora $[16,17]$.

A train of incident progressive waves of mode $p_{1}$ propagating along $x$-axis is of the form

$$
\begin{aligned}
& \phi_{0}(x, y)=e^{ \pm i p_{1} x} \cosh \left[p_{1}(H-y)\right], \quad 0 \leqslant y \leqslant H \\
& \varphi_{0}(x, y)=e^{ \pm i p_{1} x} Z\left(p_{1}, y\right), \quad-h \leqslant y \leqslant 0 .
\end{aligned}
$$

Similarly, a train of incident progressive waves of mode $\mathrm{p}_{2}$ propagating along $x$-axis is of the form

$$
\begin{aligned}
& \phi_{0}(x, y)=e^{ \pm i p_{2} x} \cosh \left[p_{2}(H-y)\right], \quad 0 \leqslant y \leqslant H \\
& \varphi_{0}(x, y)=e^{ \pm i p_{2} x} Z\left(p_{2}, y\right), \quad-h \leqslant y \leqslant 0 .
\end{aligned}
$$

When a train of incident progressive waves of a particular mode encounters a submerged or floating obstacle in an ocean, some of the energy (reflected and transmitted) from the incident wave mode is transferred to the other mode due to scattering by the obstacle. Therefore, if a wave train of mode $p_{1}$ is normally incident on the positive $\mathbf{x}$-axis on the cylindrical undulation at the bottom of a two-layer fluid, then the reflected and transmitted waves of both the modes $p_{1}$ and $p_{2}$ occur in both the layers. Now, we construct 
the far-field form of the potential functions $\phi$ and $\varphi$ by using the method of Maiti and Mandal [11] and Mohapatra and Bora [18]. Since a train of incident progressive waves of mode $p_{1}$ interacts with a small bottom undulation, the wave train is partially reflected and partially transmitted over it. In this case, the far-field behaviors of $\phi$ and $\varphi$, respectively, are

$$
\begin{aligned}
& \phi(x, y) \sim\left\{\begin{array}{l}
\cosh \left[p_{1}(H-y)\right]\left[e^{i p_{1} x}+r e^{-i p_{1} x}\right] \\
+R \cosh \left[p_{2}(H-y)\right] e^{-i p_{2} x} \text { as } x \rightarrow-\infty \\
t \cosh \left[p_{1}(H-y)\right] e^{i p_{1} x}+T \cosh \left[p_{2}(H-y)\right] e^{i p_{2} x} \quad \text { as } x \rightarrow \infty,
\end{array}\right. \\
& \varphi(x, y) \sim\left\{\begin{array}{l}
Z\left(p_{1}, y\right)\left[e^{i p_{1} x}+r e^{-i p_{1} x}\right]+R Z\left(p_{2}, y\right) e^{-i p_{2} x} \text { as } x \rightarrow-\infty, \\
t Z\left(p_{1}, y\right) e^{i p_{1} x}+T Z\left(p_{2}, y\right) e^{i p_{2} x} \text { as } x \rightarrow \infty
\end{array}\right.
\end{aligned}
$$

The unknown coefficients $r$ and $R$ are the reflection coefficients associated with wave modes $p_{1}$ and $p_{2}$, respectively, due to normal incident waves of mode $p_{1}$, and are to be determined. Similarly, $t$ and $T$ are the transmission coefficients associated with wave modes $p_{1}$ and $p_{2}$, respectively, due to normal incident waves of mode $p_{1}$.

Assuming $\epsilon$ to be very small for bottom undulation, and neglecting the second order terms, we express the boundary condition $\partial \phi / \partial \mathrm{n}=0$ on the bottom surface $y=H+\epsilon c(x)$ in an appropriate form as

$$
\frac{\partial \phi}{\partial y}-\epsilon \frac{\partial}{\partial x}\left[c(x) \frac{\partial \phi}{\partial x}\right]+\mathcal{O}\left(\epsilon^{2}\right)=0 \quad \text { on } y=H .
$$

In this case, if a train of incident progressive waves has mode $p_{1}$, then the corresponding potential functions $\phi$ and $\varphi$ satisfy the respective Laplace's equation, the free-surface condition, interface conditions, the bottom condition (17), and the far-field conditions (15) and (16) involving the unknown coefficients $r, R, t$ and $T$.

Similarly, for a wave train of mode $\mathrm{p}_{2}$ normally incident on the bottom undulation of a two-layer fluid and propagating along the positive $x$-axis, the 
far-field behaviours of $\phi$ and $\varphi$, respectively, are

$$
\begin{gathered}
\phi(x, y) \sim\left\{\begin{array}{l}
\cosh \left[p_{2}(H-y)\right]\left[e^{i p_{2} x}+R^{*} e^{-i p_{2} x}\right] \\
+r^{*} \cosh \left[p_{1}(H-y)\right] e^{-i p_{1} x} \quad \text { as } x \rightarrow-\infty, \\
T^{*} \cosh \left[p_{2}(H-y)\right] e^{i p_{2} x} \\
+t^{*} \cosh \left[p_{1}(H-y)\right] e^{i p_{1} x} \text { as } x \rightarrow \infty,
\end{array}\right. \\
\varphi(x, y) \sim\left\{\begin{array}{l}
Z\left(p_{2}, y\right)\left[e^{i p_{2} x}+R^{*} e^{-i p_{2} x}\right] \\
+r^{*} Z\left(p_{1}, y\right) e^{-i p_{1} x} \text { as } x \rightarrow-\infty, \\
T^{*} Z\left(p_{2}, y\right) e^{i p_{2} x}+t^{*} Z\left(p_{1}, y\right) e^{i p_{1} x} \text { as } x \rightarrow \infty,
\end{array}\right.
\end{gathered}
$$

where the unknown coefficients $r^{*}$ and $R^{*}$ denote the reflection coefficients associated with wave modes $p_{1}$ and $p_{2}$, respectively, due to normal incident waves of mode $p_{2}$, and are to be determined. Similarly, $t^{*}$ and $T^{*}$ denote the transmission coefficients of modes $p_{1}$ and $p_{2}$, respectively, due to normal incident waves of mode $p_{2}$. Thus, if a train of incident waves has mode $p_{2}$, then it satisfies the respective Laplace's equation, the free-surface condition, interface conditions and the bottom condition (17). Also $\phi$ and $\varphi$ satisfy the far-field conditions (18) and (19) involving the unknown coefficients $r^{*}, \mathbf{R}^{*}, \mathbf{t}^{*}$ and $\mathrm{T}^{*}$.

\section{Method of solution}

Since there are two modes of wave propagating along the positive $x$-direction in a two-layer fluid region, for each wave mode we calculate the first-order reflection and transmission coefficients associated with respective mode of wave by the following procedure. 


\subsection{Perturbation technique}

As the lower layer fluid has a small bottom undulation, we approximate the normal velocity potential in terms of a series involving powers of a small parameter $\epsilon$. This provides a clue for a perturbation analysis in which the velocity potentials and the reflection and transmission coefficients appearing in the coupled boundary value problem (BVP) are expressed as a power series involving this parameter $\epsilon$. After equating the coefficients of identical powers of the parameter $\epsilon$ on both sides of all the equations and the conditions of the BVP, several BVPs are obtained. The BVP for the first-order is only solved here. Here, the small parameter $\epsilon$ is different from the wave steepness used by Chapman and Porter [2] in their investigation of free sloshing of an inviscid fluid over an arbitrary bed.

Let us first consider a train of progressive waves of mode $p_{1}$ to be normally incident on the bottom undulation. In the absence of any undulation at the bottom, a train of normal incident waves propagates without any hindrance resulting in transmission only. In view of this and the appropriate form of the boundary condition (17), we assume a perturbation expansion for $\phi, \varphi, r, \mathrm{R}, \mathrm{t}$ and $\mathrm{T}$ in terms of $\epsilon$ as

$$
\left\{\begin{array}{l}
\phi=\phi_{0}+\epsilon \phi_{1}+\mathcal{O}\left(\epsilon^{2}\right) \\
\varphi=\varphi_{0}+\epsilon \varphi_{1}+\mathcal{O}\left(\epsilon^{2}\right) \\
r=\epsilon r_{1}+\mathcal{O}\left(\epsilon^{2}\right) \\
R=\epsilon R_{1}+\mathcal{O}\left(\epsilon^{2}\right) \\
t=1+\epsilon t_{1}+\mathcal{O}\left(\epsilon^{2}\right) \\
T=\epsilon T_{1}+\mathcal{O}\left(\epsilon^{2}\right)
\end{array}\right.
$$

where $\phi_{0}$ and $\varphi_{0}$ are given by equations (11) and (12) respectively.

Such a perturbation expansion ceases to be valid at Bragg resonance when the reflection coefficient becomes much larger than the undulation parameter $\epsilon$, as pointed out by Mei [13]. Bragg resonance occurs when the ripple wavenumber of the bottom undulation is twice the incident waves wavenumber. However, 
to overcome this Bragg resonance situation, Mei [13] developed wave evolution and reflection theory at and near Bragg resonance condition for shore-parallel sinusoidal bars. Since Bragg resonance is not taken into account in our study, the perturbation expansion given in (20) is valid throughout our work.

Using equation (20) in equations (1), (2) and boundary conditions (17), (4-6), (15) and (16), and then comparing the first-order terms of $\epsilon$ on both sides of the equations, we find that the first-order potentials $\phi_{1}(x, y)$ and $\varphi_{1}(x, y)$ satisfy the coupled boundary value problem

$$
\begin{aligned}
& \nabla^{2} \phi_{1}=0 \quad \text { in } 0 \leqslant y \leqslant H, \\
& \nabla^{2} \varphi_{1}=0 \text { in }-\mathrm{h} \leqslant \mathrm{y} \leqslant 0 \text {, } \\
& \frac{\partial \phi_{1}}{\partial y}=\mathfrak{i p} p_{1} \frac{d}{d x}\left[c(x) e^{i p_{1} x}\right] \equiv p(x) \quad \text { on } y=H \text {, } \\
& \frac{\partial \phi_{1}}{\partial y}=\frac{\partial \varphi_{1}}{\partial y} \quad \text { on } y=0 \text {, } \\
& \mathrm{K} \phi_{1}+\frac{\partial \phi_{1}}{\partial y}=\rho\left(K \varphi_{1}+\frac{\partial \varphi_{1}}{\partial y}\right) \quad \text { on } y=0 \\
& \mathrm{~K} \varphi_{1}+\frac{\partial \varphi_{1}}{\partial y}+\mathrm{S} \frac{\partial^{3} \varphi_{1}}{\partial \mathrm{y}^{3}}=0 \quad \text { on } \mathrm{y}=-\mathrm{h} \\
& \phi_{1}(x, y) \sim\left\{\begin{array}{l}
r_{1} \cosh \left[p_{1}(H-y)\right] e^{-i p_{1} x} \\
+R_{1} \cosh \left[p_{2}(H-y)\right] e^{-i p_{2} x} \quad \text { as } x \rightarrow-\infty, \\
t_{1} \cosh \left[p_{1}(H-y)\right] e^{i p_{1} x} \\
+T_{1} \cosh \left[p_{2}(H-y)\right] e^{i p_{2} x} \quad \text { as } x \rightarrow \infty,
\end{array}\right. \\
& \varphi_{1}(x, y) \sim\left\{\begin{array}{l}
r_{1} Z\left(p_{1}, y\right) e^{-i p_{1} x}+R_{1} Z\left(p_{2}, y\right) e^{-i p_{2} x} \quad \text { as } x \rightarrow-\infty \\
t_{1} Z\left(p_{1}, y\right) e^{i p_{1} x}+T_{1} Z\left(p_{2}, y\right) e^{i p_{2} x} \quad \text { as } x \rightarrow \infty
\end{array}\right.
\end{aligned}
$$

To solve the above coupled boundary value problem described by equations (21-28), we need two-dimensional source potentials (in terms of Green's function) for Laplace's equation due to a source submerged in either of the two layers. By employing Green's integral theorem, we obtain the first-order coefficients $r_{1}, R_{1}, t_{1}$ and $T_{1}$ in terms of integrals involving the shape function $c(x)$ 
(Mohapatra and Bora [18]). When the source is submerged in the lower fluid at $(\xi, \eta), 0<\eta<H$, then we consider $\mathrm{G}_{1}(x, y ; \xi, \eta)$ and $\mathrm{G}_{2}(x, y ; \xi, \eta)$ to be the source potentials in terms of Green's function for the lower and upper layer fluids, respectively. Similarly, when the source is submerged in the upper layer fluid at $(\xi, \eta),-h<\eta<0$, then we consider $G_{3}(x, y ; \xi, \eta)$ and $\mathrm{G}_{4}(x, y ; \xi, \eta)$ to be the source potentials in terms of Green's function for the lower and upper layer fluids, respectively.

\subsection{Introduction of Green's functions}

Suppose the source is submerged in the lower layer fluid. Then, for $0<$ $\eta<H$, the source potentials in terms of Green's function $G_{1}(x, y ; \xi, \eta)$ and $\mathrm{G}_{2}(x, y ; \xi, \eta)$ satisfy the following boundary value problem

$$
\begin{aligned}
& \nabla^{2} \mathrm{G}_{1}=0 \quad \text { in } 0<\mathrm{y}<\mathrm{H}, \quad \text { except at }(\xi, \eta), \\
& \nabla^{2} \mathrm{G}_{2}=0 \quad \text { in }-\mathrm{h} \leqslant \mathrm{y} \leqslant 0, \\
& \frac{\partial \mathrm{G}_{1}}{\partial \mathrm{y}}=0 \quad \text { on } \mathrm{y}=\mathrm{H}, \\
& \frac{\partial \mathrm{G}_{1}}{\partial \mathrm{y}}=\frac{\partial \mathrm{G}_{2}}{\partial \mathrm{y}} \quad \text { on } \mathrm{y}=0, \\
& \mathrm{KG}_{1}+\frac{\partial \mathrm{G}_{1}}{\partial \mathrm{y}}=\rho\left(\mathrm{KG}_{2}+\frac{\partial \mathrm{G}_{2}}{\partial \mathrm{y}}\right) \quad \text { on } \mathrm{y}=0, \\
& \mathrm{KG}_{2}+\frac{\partial \mathrm{G}_{2}}{\partial \mathrm{y}}+\mathrm{S} \frac{\partial^{3} \mathrm{G}_{2}}{\partial \mathrm{y}^{3}}=0 \quad \text { on } \mathrm{y}=-\mathrm{h}, \\
& \mathrm{G}_{1} \sim \log \mathrm{r} \text { as } \mathrm{r}=\sqrt{(x-\xi)^{2}+(\mathrm{y}-\eta)^{2}} \rightarrow 0 .
\end{aligned}
$$

Here, both $G_{1}$ and $G_{2}$ represent outgoing waves as $|x-\xi| \rightarrow \infty$. Velocity potentials due to various types of singularities of water waves are generally termed as source potentials and have wide applications in the linearized theory of water waves (Mohapatra and Bora [18]). If a body in water undergoes some sort of oscillations, then we express the resulting motion in the fluid by a 
series of singularities placed in the body. These singularities are characterized by their giving rise to velocity potentials, which are typical singular solutions of the Laplace's equation in the neighborhood of the singularity. For the two-dimensional case, these singularities are logarithmic, and for the threedimensional case, these are point sources or point multipoles.

In order that the boundary conditions (29-35) are satisfied, the above source potentials $\mathrm{G}_{1}(x, y ; \xi, \eta)$ and $\mathrm{G}_{2}(x, y ; \xi, \eta)$ are

$$
\begin{aligned}
& G_{1}(x, y ; \xi, \eta)=-2 \pi i K\left[\frac{Y_{1}\left(p_{1}\right) \cosh \left[p_{1}(H-\eta)\right] \cosh \left[p_{1}(H-y)\right]}{p_{1} \sinh p_{1} H \Delta^{\prime}\left(p_{1}\right)} e^{i p_{1}|x-\xi|}\right. \\
& \left.\quad+\frac{Y_{1}\left(p_{2}\right) \cosh \left[p_{2}(H-\eta)\right] \cosh \left[p_{2}(H-y)\right]}{p_{2} \sinh p_{2} H \Delta^{\prime}\left(p_{2}\right)} e^{i p_{2}|x-\xi|}\right] \\
& +2 \pi K \sum_{n=1}^{\infty} \frac{\left[K \cos \kappa_{n} h+\left(1-S \kappa_{n}^{2}\right) \kappa_{n} \sin \kappa_{n} h\right] \cos \kappa_{n}(H-\eta) \cos \kappa_{n}(H-y)}{\kappa_{n} \sin \kappa_{n} H \Delta^{\prime}\left(i \kappa_{n}\right)} \\
& \quad \times e^{-\kappa_{n}|x-\xi|}, \\
& G_{2}(x, y ; \xi, \eta)=-2 \pi i K\left[\frac{Y_{2}\left(p_{1}\right) \cosh \left[p_{1}(H-\eta)\right]}{p_{1} \Delta^{\prime}\left(p_{1}\right)} e^{i p_{1}|x-\xi|}\right. \\
& \left.\quad+\frac{Y_{2}\left(p_{2}\right) \cosh \left[p_{2}(H-\eta)\right]}{p_{2} \Delta^{\prime}\left(p_{2}\right)} e^{i p_{2}|x-\xi|}\right] \\
& -2 \pi K \sum_{n=1}^{\infty} \frac{\left[\left(1-S \kappa_{n}^{2}\right) \kappa_{n} \cos \kappa_{n}(h+y)-K \sin \kappa_{n}(h+y)\right] \cos \kappa_{n}(H-\eta)}{\kappa_{n} \Delta^{\prime}\left(i \kappa_{n}\right)} \\
& \quad \times e^{-\kappa_{n}|x-\xi|},
\end{aligned}
$$

where

$$
\begin{aligned}
& Y_{1}(u)=K \cosh u h-\left(1+S u^{2}\right) u \sinh u h, \quad u=p_{1}, p_{2}, \\
& Y_{2}(u)=\left(1+S u^{2}\right) u \cosh [u(h+y)]-K \sinh [u(h+y)], \quad u=p_{1}, p_{2},
\end{aligned}
$$

and $\Delta$ is given in equation (10) with $\Delta^{\prime}$ denoting the derivative of $\Delta$ with respect to $\mathrm{u}$. In the above equations, $\boldsymbol{\kappa}_{\mathrm{n}}$ 's are real and positive, satisfying 
the following equation in $\mathrm{k}$ :

$$
\begin{aligned}
& K^{2} \cos \kappa h \cos \kappa H-\left[K^{2} \rho-\kappa^{2}(1-\rho)\left(1-S \kappa^{2}\right)\right] \sin \kappa h \sin \kappa H \\
& +K \kappa\left[\left(1-S \kappa^{2}\right) \sin \kappa h \cos \kappa H+\left(1-\rho S \kappa^{2}\right) \sin \kappa H \cos \kappa h\right]=0 .
\end{aligned}
$$

The summation term in each of (36) and (37) corresponds to evanescent modes propagating along the positive $x$-direction in a two-layer fluid. The solutions $\mathrm{G}_{1}(x, y ; \xi, \eta)$ and $\mathrm{G}_{2}(x, y ; \xi, \eta)$, as $|x-\xi| \rightarrow \infty$, are

$$
\begin{aligned}
G_{1}(x, y ; \xi, \eta)= & -2 \pi i K\left\{\frac{Y_{1}\left(p_{1}\right) \cosh \left[p_{1}(H-\eta)\right] \cosh \left[p_{1}(H-y)\right]}{p_{1} \sinh p_{1} H \Delta^{\prime}\left(p_{1}\right)} e^{i p_{1}|x-\xi|}\right. \\
& \left.+\frac{Y_{1}\left(p_{2}\right) \cosh \left[p_{2}(H-\eta)\right] \cosh \left[p_{2}(H-y)\right]}{p_{2} \sinh p_{2} H \Delta^{\prime}\left(p_{2}\right)} e^{i p_{2}|x-\xi|}\right\}, \\
G_{2}(x, y ; \xi, \eta)= & -2 \pi i K\left\{\frac{Y_{2}\left(p_{1}\right) \cosh \left[p_{1}(H-\eta)\right]}{p_{1} \Delta^{\prime}\left(p_{1}\right)} e^{i p_{1}|x-\xi|}\right. \\
& \left.+\frac{Y_{2}\left(p_{2}\right) \cosh \left[p_{2}(H-\eta)\right]}{p_{2} \Delta^{\prime}\left(p_{2}\right)} e^{i p_{2}|x-\xi|}\right\}
\end{aligned}
$$

Similarly, when the source term $(\xi, \eta),-h<\eta<0$, is submerged in the upper layer fluid, then the source potentials $\mathrm{G}_{3}(x, y ; \xi, \eta)$ and $\mathrm{G}_{4}(x, y ; \xi, \eta)$ satisfy the same type of boundary value problem, as given by equations (29-34), with $\mathrm{G}_{1}$ replaced by $\mathrm{G}_{3}$ and $\mathrm{G}_{2}$ by $\mathrm{G}_{4}$, and additionally $\mathrm{G}_{4}$ satisfies

$$
\mathrm{G}_{4} \sim \log \mathrm{r}^{\prime} \quad \text { as } \mathrm{r}^{\prime}=\sqrt{(\mathrm{x}-\xi)^{2}+(\mathrm{y}+\eta)^{2}} \rightarrow 0 .
$$

Here, $\mathrm{G}_{3}$ and $\mathrm{G}_{4}$ also represent outgoing waves as $|x-\xi| \rightarrow \infty$. The source 
potentials $\mathrm{G}_{3}(x, y ; \xi, \eta)$ and $\mathrm{G}_{4}(x, y ; \xi, \eta)$, as $|x-\xi| \rightarrow \infty$, are

$$
\begin{aligned}
\mathrm{G}_{3}(x, y ; \xi, \eta)= & 2 \pi i \rho K\left\{\frac{Y_{3}\left(p_{1}\right) \cosh \left[p_{1}(H-y)\right]}{p_{1} \Delta^{\prime}\left(p_{1}\right)} e^{i p_{1}|x-\xi|}\right. \\
& \left.+\frac{Y_{3}\left(p_{2}\right) \cosh \left[p_{2}(H-y)\right]}{p_{2} \Delta^{\prime}\left(p_{2}\right)} e^{i p_{2}|x-\xi|}\right\}, \\
G_{4}(x, y ; \xi, \eta)= & 2 \pi i \rho K\left[\frac{Y_{2}\left(p_{1}\right) Y_{3}\left(p_{1}\right) \sinh p_{1} H}{p_{1} \Delta^{\prime}\left(p_{1}\right) Y_{1}\left(p_{1}\right)} e^{i p_{1}|x-\xi|}\right. \\
& \left.+\frac{Y_{2}\left(p_{2}\right) Y_{3}\left(p_{2}\right) \sinh p_{2} H}{p_{2} \Delta^{\prime}\left(p_{2}\right) Y_{1}\left(p_{2}\right)} e^{i p_{2}|x-\xi|}\right],
\end{aligned}
$$

where

$$
\mathrm{Y}_{3}(\mathfrak{u})=K \sinh [u(h+\eta)]-\left(1+S \mathfrak{u}^{2}\right) \mathfrak{u} \cosh [u(h+\eta)], \quad u=p_{1}, p_{2} .
$$

If the surface tension on the free-surface is assumed to be negligible (implying $S \rightarrow 0$ ), then the representation of the source potentials, as $|x-\xi| \rightarrow \infty$, given by equations (38-41), coincide with the corresponding source potentials obtained in the normal case where the two-layer fluid has a free-surface (effect of surface tension neglected) and a bottom undulation (Maiti and Mandal [11]).

To calculate $\phi_{1}(\xi, \eta)$, when the source term $(\xi, \eta), 0<\eta<H$, is submerged in the lower layer fluid, we apply Green's integral theorem to $\phi_{1}(x, y)$ and $\mathrm{G}_{1}(x, y ; \xi, \eta)$ in the form

$$
\int_{C}\left(\phi_{1} \frac{\partial G_{1}}{\partial n}-G_{1} \frac{\partial \phi_{1}}{\partial n}\right) d s=0
$$

where $C$ is a closed contour in the $x y$-plane consisting of the lines $y=0$, $-X \leqslant x \leqslant X ; y=H,-X \leqslant x \leqslant X ; x= \pm X, 0 \leqslant y \leqslant H$ and a small circle of radius $\gamma$ with center at $(\xi, \eta)$ and ultimately letting $X \rightarrow \infty$ and $\gamma \rightarrow 0$. Then there will be no contribution to the integrals from the lines $x= \pm X$, as both $\phi_{1}$ and $G_{1}$ approach zero when $X \rightarrow \pm \infty$. Thus the resultant form of 
the integral equation (42) is

$-2 \pi \phi_{1}(\xi, \eta)+\int_{-\infty}^{\infty} p(x) G_{1}(x, H ; \xi, \eta) d x+\int_{-\infty}^{\infty}\left(\phi_{1} \frac{\partial G_{1}}{\partial y}-G_{1} \frac{\partial \phi_{1}}{\partial y}\right)_{y=0} d x=0$

Then again, we apply Green's integral theorem to $\varphi_{1}(x, y)$ and $G_{2}(x, y ; \xi, \eta)$ in the form

$$
\int_{C^{\prime}}\left(\varphi_{1} \frac{\partial G_{2}}{\partial \mathrm{n}}-\mathrm{G}_{2} \frac{\partial \varphi_{1}}{\partial \mathrm{n}}\right) \mathrm{ds}=0
$$

where $C^{\prime}$ is a closed counter consisting of the lines $y=-h,-X \leqslant x \leqslant X$; $y=0,-X \leqslant x \leqslant X ; x= \pm X,-h \leqslant y \leqslant 0$ and ultimately letting $X \rightarrow \infty$. Here, $G_{2}(x, y ; \xi, \eta)$ has no singularity in the upper region. Now rewrite the equation (44) as

$$
\begin{aligned}
& -\int_{-\infty}^{\infty}\left(\varphi_{1} \frac{\partial G_{2}}{\partial y}-G_{2} \frac{\partial \varphi_{1}}{\partial y}\right)_{y=-h} d x-\int_{-h}^{0}\left(\varphi_{1} \frac{\partial G_{2}}{\partial y}-G_{2} \frac{\partial \varphi_{1}}{\partial y}\right)_{x=-\infty} d y \\
& +\int_{-\infty}^{\infty}\left(\varphi_{1} \frac{\partial G_{2}}{\partial y}-G_{2} \frac{\partial \varphi_{1}}{\partial y}\right)_{y=0} d x+\int_{-h}^{0}\left(\varphi_{1} \frac{\partial G_{2}}{\partial y}-G_{2} \frac{\partial \varphi_{1}}{\partial y}\right)_{x=\infty} d y=0 .
\end{aligned}
$$

Use of the free-surface condition on $y=-h$ and the asymptotic results for $\varphi_{1}$ and $G_{2}$, make the first integral equal to zero for any large value of $X$. The second and fourth integrals are zero when $X \rightarrow \infty$, due to the outgoing nature of both $\varphi_{1}$ and $G_{2}$ as $x \rightarrow \pm \infty$. Thus the resultant integral of (45) is

$$
\int_{-\infty}^{\infty}\left(\varphi_{1} \frac{\partial G_{2}}{\partial y}-G_{2} \frac{\partial \varphi_{1}}{\partial y}\right)_{y=0} d x=0 .
$$

Now solving equations (43) and (46) with the help of interface conditions at $y=0$ (i.e., the conditions (24) and (25) are the interface conditions for $\phi_{1}$ and $\varphi_{1}$, and the conditions (32) and (33) are the interface conditions for $\mathrm{G}_{1}$ and $\mathrm{G}_{2}$ ), we get

$$
\phi_{1}(\xi, \eta)=\frac{1}{2 \pi} \int_{-\infty}^{\infty} G_{1}(x, H ; \xi, \eta) p(x) d x \quad 0<\eta<H,
$$


which solves the boundary value problem for $\phi_{1}(x, y)$.

Similarly, to calculate $\varphi_{1}(\xi, \eta)$, when the source term $(\xi, \eta),-h<\eta<0$, is submerged in the upper layer fluid, we apply the same procedure as followed previously in the case when the source term was submerged in the lower fluid. The final expression for $\varphi_{1}(\xi, \eta)$ is

$$
\varphi_{1}(\xi, \eta)=\frac{1}{2 \pi \rho} \int_{-\infty}^{\infty} G_{3}(x, H ; \xi, \eta) p(x) d x \quad-h<\eta<0,
$$

which solves the boundary value problem for $\varphi_{1}(x, y)$.

\section{Reflection and transmission coefficients}

The first-order reflection and transmission coefficients $r_{1}, t_{1}$ and $R_{1}, T_{1}$ are now obtained by letting $\xi \rightarrow-\infty$ and $\xi \rightarrow \infty$, respectively, in equation (47) and comparing with equation $(27)$ by replacing $(\chi, y)$ with $(\xi, \eta)$. Thus we obtain $r_{1}$ and $R_{1}$ as

$$
\begin{aligned}
& r_{1}=-\frac{i K p_{1} Y_{1}\left(p_{1}\right)}{\sinh p_{1} H \Delta^{\prime}\left(p_{1}\right)} \int_{-\infty}^{\infty} e^{2 i p_{1} x} c(x) d x \\
& R_{1}=-\frac{i K p_{1} Y_{1}\left(p_{2}\right)}{\sinh p_{2} H \Delta^{\prime}\left(p_{2}\right)} \int_{-\infty}^{\infty} e^{i\left(p_{1}+p_{2}\right) x} c(x) d x .
\end{aligned}
$$

Similarly, to find $t_{1}$ and $T_{1}$, we consider $\phi_{1}(x, y)$ from the equation (27) by replacing $(x, y)$ with $(\xi, \eta)$ and $G_{1}(x, y ; \xi, \eta)$ from the equation (38) with $\xi \rightarrow \infty$, and use them in equation (47) to get

$$
\begin{aligned}
t_{1} & =\frac{i K p_{1} Y_{1}\left(p_{1}\right)}{\sinh p_{1} H \Delta^{\prime}\left(p_{1}\right)} \int_{-\infty}^{\infty} c(x) d x \\
T_{1} & =\frac{i K p_{1} Y_{1}\left(p_{2}\right)}{\sinh p_{2} H \Delta^{\prime}\left(p_{2}\right)} \int_{-\infty}^{\infty} e^{i\left(p_{1}-p_{2}\right) x} c(x) d x
\end{aligned}
$$


The same expressions for $r_{1}, R_{1}, t_{1}$ and $T_{1}$ are also obtained by letting $\xi \rightarrow$ $-\infty$ and $\xi \rightarrow \infty$, respectively, in equations (28) and (40), and solving for equation (48). Therefore, the two first-order reflection coefficients and the two first-order transmission coefficients can be evaluated from equations (49-52) once the shape function $c(x)$ is known.

When we consider a train of progressive waves of mode $p_{2}$ to be normally incident on the bottom undulation, the same mathematical procedure described above for the case of mode $p_{1}$ is followed in obtaining the first-order reflection and transmission coefficients $r_{1}^{*}, R_{1}^{*}, t_{1}^{*}$ and $T_{1}^{*}$. The final expressions for this case are

$$
\begin{aligned}
r_{1}^{*} & =-\frac{i K p_{2} Y_{1}\left(p_{1}\right)}{\sinh p_{1} H \Delta^{\prime}\left(p_{1}\right)} \int_{-\infty}^{\infty} e^{i\left(p_{1}+p_{2}\right) x} c(x) d x, \\
R_{1}^{*} & =-\frac{i K p_{2} Y_{1}\left(p_{2}\right)}{\sinh p_{2} H \Delta^{\prime}\left(p_{2}\right)} \int_{-\infty}^{\infty} e^{2 i p_{2} x} c(x) d x \\
t_{1}^{*} & =\frac{i K p_{2} Y_{1}\left(p_{1}\right)}{\sinh p_{1} H \Delta^{\prime}\left(p_{1}\right)} \int_{-\infty}^{\infty} e^{i\left(p_{2}-p_{1}\right) x} c(x) d x \\
T_{1}^{*} & =\frac{i K p_{2} Y_{1}\left(p_{2}\right)}{\sinh p_{2} H \Delta^{\prime}\left(p_{2}\right)} \int_{-\infty}^{\infty} c(x) d x
\end{aligned}
$$

Here, if we take $S=0$ (i.e., the surface tension on the free-surface is assumed to be zero), then the results of Maiti and Mandal [11] are recovered from (4956). Moreover, all the above results obtained here in the absence of surface tension coincide with the corresponding results of Mohapatra and Bora [18] for the case of free-surface.

The following section examine the effect of surface tension on the reflection and transmission coefficients for a special sinusoidal form of the shape function $c(x)$ on the bottom undulation. 


\section{Special form of the bottom topography}

Here, we consider a special sinusoidal form of the shape function $c(x)$ for an uneven bottom surface. This functional form of the bottom deformation closely resembles some naturally occurring obstacles formed at the bottom due to sedimentation and ripple in sands. Davies [3] studied sinusoidal form of bottom undulation on an ocean bed for a single-layer fluid by using a Fourier transform and found that an undulating bed has the ability to reflect incident wave energy which has important implications in respect of coastal protection as well as possible ripple growth if the bed is erodible. Because of the importance of the bed topographies with sinusoidal ripples in applications, significant emphasis is laid upon them.

The shape function $c(x)$ in the form of a patch of sinusoidal bottom ripples on the bottom surface with amplitude $a$ on an otherwise flat bottom has the form

$$
c(x)=\left\{\begin{array}{l}
a \sin l x, \quad-L \leqslant x \leqslant L, \\
0, \quad \text { otherwise }
\end{array}\right.
$$

Here, $L=n \pi / l$, where $n$ is a positive integer which denotes the number of ripples, and $l$ is the wavenumber of the patch in the region $-\mathrm{L} \leqslant \mathrm{x} \leqslant \mathrm{L}$. For this particular choice of the domain $[-\mathrm{L}, \mathrm{L}]$ for the shape function $\mathrm{c}(\mathrm{x})$, the transmission coefficients $t_{1}$ and $T_{1}^{*}$ vanish identically (because $c(x)$ is an odd function).

The first-order reflection and transmission coefficients $r_{1}, R_{1}$ and $T_{1}$ with respect to the modes $p_{1}$ and $p_{2}$, respectively, due to normal incident waves of mode $p_{1}$, are obtained by substituting the value of $c(x)$ from equation (57) 
into equations (49), (50) and (52). These coefficients are

$$
\begin{aligned}
& \mathrm{r}_{1}=(-1)^{\mathrm{n}} 2 a l K p_{1} W_{1}\left[\frac{\sin \left(2 n \pi p_{1} / l\right)}{l^{2}-4 p_{1}^{2}}\right], \\
& \mathrm{R}_{1}=(-1)^{\mathrm{n}} 2 a l K p_{1} W_{2}\left\{\frac{\sin \left[n \pi\left(p_{1}+p_{2}\right) / l\right]}{l^{2}-\left(p_{1}+p_{2}\right)^{2}}\right\}, \\
& \mathrm{T}_{1}=(-1)^{\mathrm{n}+1} 2 a l K p_{1} W_{2}\left\{\frac{\sin \left[n \pi\left(p_{1}-p_{2}\right) / l\right]}{l^{2}-\left(p_{1}-p_{2}\right)^{2}}\right\},
\end{aligned}
$$

where

$$
W_{j}=\frac{\left(1+S p_{j}^{2}\right) p_{j} \sinh p_{j} h-K \cosh p_{j} h}{\sinh p_{j} H \Delta^{\prime}\left(p_{j}\right)}, \quad j=1,2 .
$$

Similarly, the reflection and transmission coefficients $r_{1}^{*}, R_{1}^{*}$ and $t_{1}^{*}$ with respect to the modes $p_{1}$ and $p_{2}$, respectively, due to normal incident waves of mode $\mathrm{p}_{2}$, are obtained by substituting the value of $\mathrm{c}(\mathrm{x})$ from equation (57) into equations (53-55). These coefficients are

$$
\begin{aligned}
& r_{1}^{*}=(-1)^{n} 2 a l K p_{2} W_{1}\left\{\frac{\sin \left[n \pi\left(p_{2}+p_{1}\right) / l\right]}{l^{2}-\left(p_{2}+p_{1}\right)^{2}}\right\}, \\
& R_{1}^{*}=(-1)^{n} 2 a l K p_{2} W_{2}\left[\frac{\sin \left(2 n p_{2} \pi / l\right)}{l^{2}-4 p_{2}^{2}}\right], \\
& t_{1}^{*}=(-1)^{n+1} 2 a l K p_{2} W_{1}\left\{\frac{\sin \left[n \pi\left(p_{2}-p_{1}\right) / l\right]}{l^{2}-\left(p_{2}-p_{1}\right)^{2}}\right\} .
\end{aligned}
$$

The relations (58-60) and (62-64) show that for a given number of ripples $\mathfrak{n}$, the first-order reflection and transmission coefficients are oscillatory in nature. Further, equation (58) shows that when the incident waves of mode $p_{1}$ and the ripples wavenumber $l$ of the undulating bed satisfy $l=2 p_{1}$, the theory predicts the resonant interaction between the incident waves and the sinusoidal ripples (Mei [13]). Similarly, when $l=p_{1}+p_{2}$ or $l=p_{1}-p_{2}$, the theory also predicts the resonance interaction between the incident waves and the sinusoidal ripples. In a similar manner, we investigate the resonance phenomena for the case of a train of incident waves of mode $p_{2}$. Further, the 
relations (58-60) and (62-64) show that the reflection as well as transmission coefficients also depends on the surface tension parameter. To study the effect of the various parameters (the surface tension parameters, the total number of ripples and the density ratios) on the reflected and transmitted wave energy, the numerical computation of the reflection and transmission coefficients given by (58-60) and (62-64) is discussed in the next section.

\section{$6 \quad$ Numerical results}

Figures 2-10 show the first-order reflection and transmission coefficients for the case of a train of normal incident waves of wavenumber $p_{1}$ (free-surface wave mode) propagating in the positive $x$-direction over a small bottom undulation. In all the figures, the depth ratio $\mathrm{H} / \mathrm{h}$ is taken as 2 , the amplitude of the sinusoidal ripples $\mathrm{a} / \mathrm{h}$ as 0.1 and the ripple wavenumber as $l \mathrm{~h}=1$. For a two-layer fluid consisting of fresh water and salt water, the value of $\rho$ would ideally be around 0.97 . We report on the density ratios as $\rho=0.5,0.6$ and 0.9 instead of a realistic ratio such as 0.97 .

1. If the case of the ratio of the densities near to one, computationally the values of the two roots of the dispersion relation are very near to each other. In this case the two-layer fluid behaves like a single-layer fluid. For a single-layer fluid, only one non-zero root exists indicating the wavenumber of the fluid region. However, for a given frequency in a two-layer fluid bounded above by a free-surface, there exist two non-zero roots of the dispersion equation. The entire analytical study for the problem of this type of two-layer fluid of finite depth is rendered inappropriate unless two wavenumbers exist.

2. To avoid the above mentioned numerical difficulties and to get a clear observation of the reflection and transmission coefficients corresponding to each wavenumber, we consider the ratio of the densities as $\rho=0.5$, 0.6 and 0.9. Also, we have found that others (Linton and McIver [9], 
Figure 2: Reflection coefficient $\left|r_{1}\right|$ due to wave of mode $p_{1}$ for $\rho=0.5$ and $\mathrm{n}=3$.

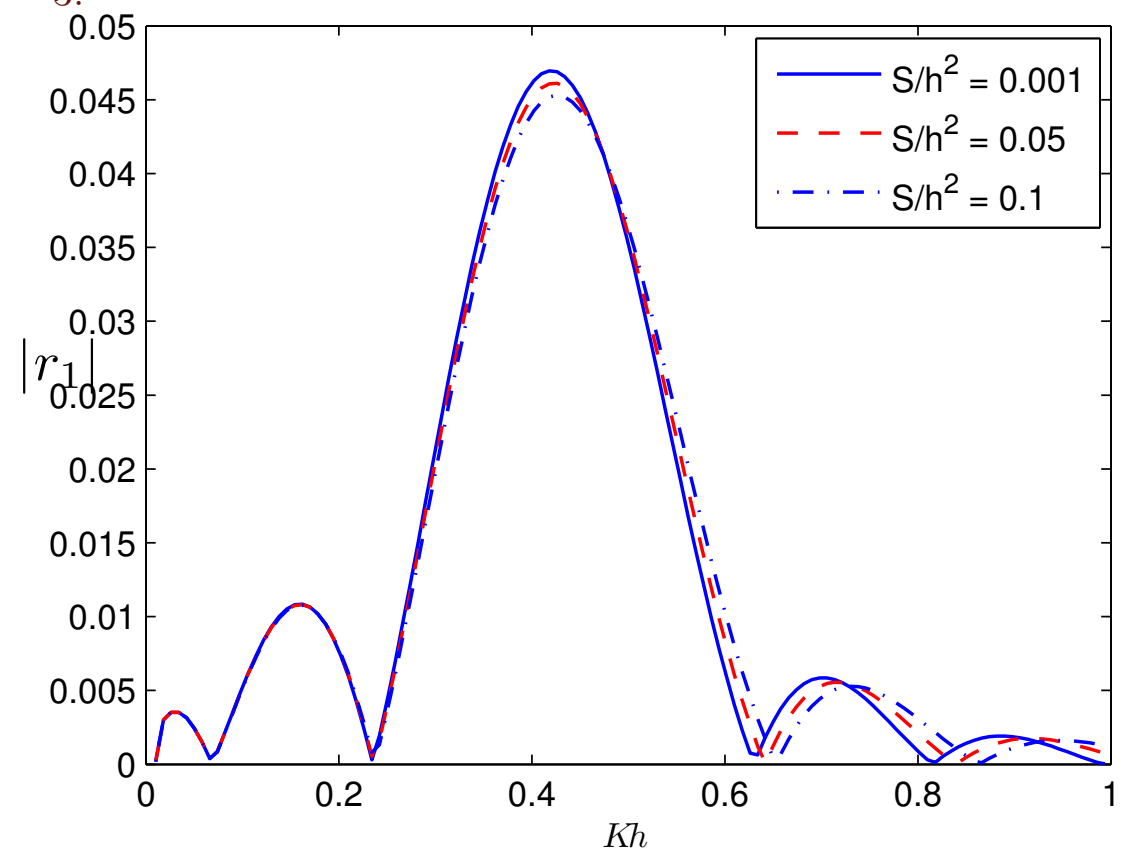

Maiti and Mandal [11]) used such density ratios while dealing with two-layer fluid problems.

The different curves in Figures 2-4 correspond to three different surface tension parameters $S / h^{2}=0.001,0.05$ and 0.1 , while the number of ripples $n$ is fixed at 3 , and the density ratio $\rho$ is taken as 0.5 . The peak values of the reflection coefficient $\left|r_{1}\right|$ of waves with wavenumber $p_{1}$ for normal incident waves of wavenumber $p_{1}$, shown in Figure 2 decrease as the surface tension parameter $\mathrm{S} / \mathrm{h}^{2}$ increases. Figures 3 and 4 , respectively, show the firstorder reflection and transmission coefficients of the waves of wavenumber $p_{2}$ due to the normal incident waves of wavenumber $p_{1}$ and show that as the values of surface tension parameter increase, the peak values of $\left|R_{1}\right|$ and $\left|T_{1}\right|$ increase but their non-zero values show that some conversion of energy from 
Figure 3: Reflection coefficient $\left|R_{1}\right|$ due to wave of mode $p_{1}$ for $\rho=0.5$ and $\mathrm{n}=3$.

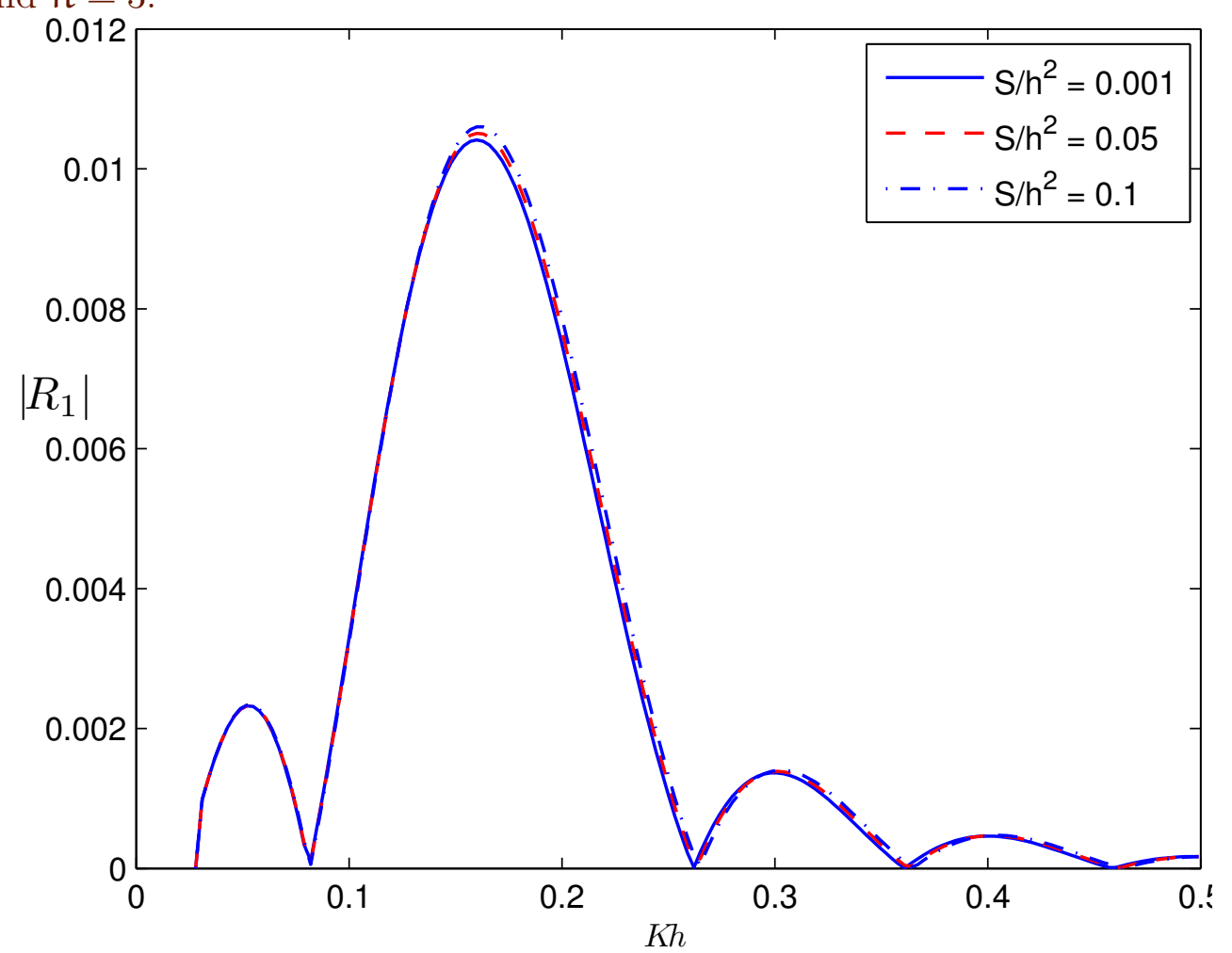

one wavenumber to the other is always possible. Moreover, the reflection coefficient of the waves of wavenumber $p_{2}$ is found to be smaller in comparison to those of the waves with wavenumber $p_{1}$. All the figures show that when the values of surface tension parameter $S / h^{2}$ increase, the rate of change of the values of reflection and transmission coefficients for the waves with wavenumber $p_{2}$ due to the normal incident waves of wavenumber $p_{1}$ is smaller than in the case of $\left|r_{1}\right|$. 
Figure 4: Transmission coefficient $\left|T_{1}\right|$ due to wave of mode $p_{1}$ for $\rho=0.5$ and $\mathrm{n}=3$.

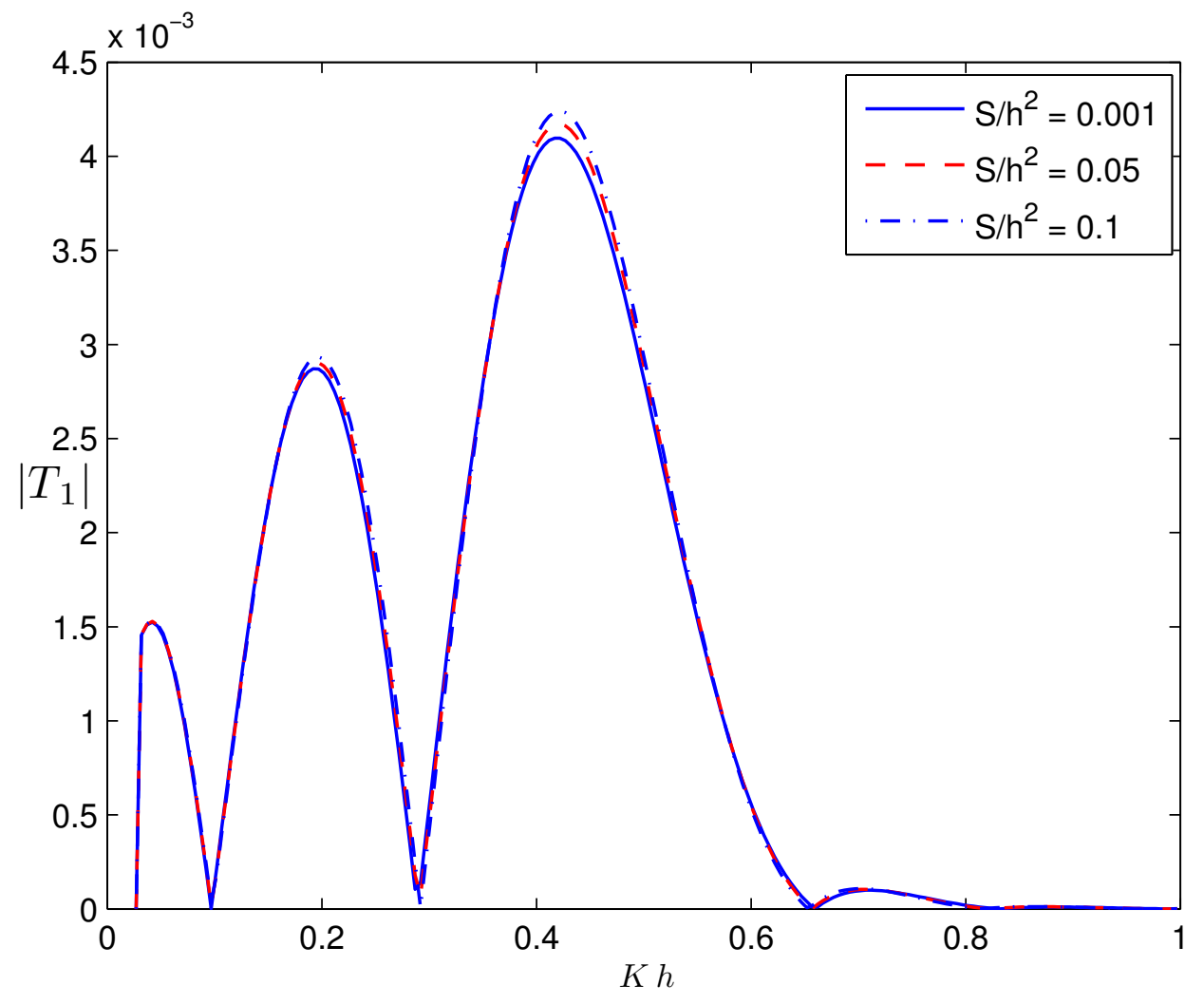


Figure 5: Reflection coefficient $\left|r_{1}\right|$ due to wave of mode $p_{1}$ for $S / h^{2}=0.001$ and $\rho=0.5$.

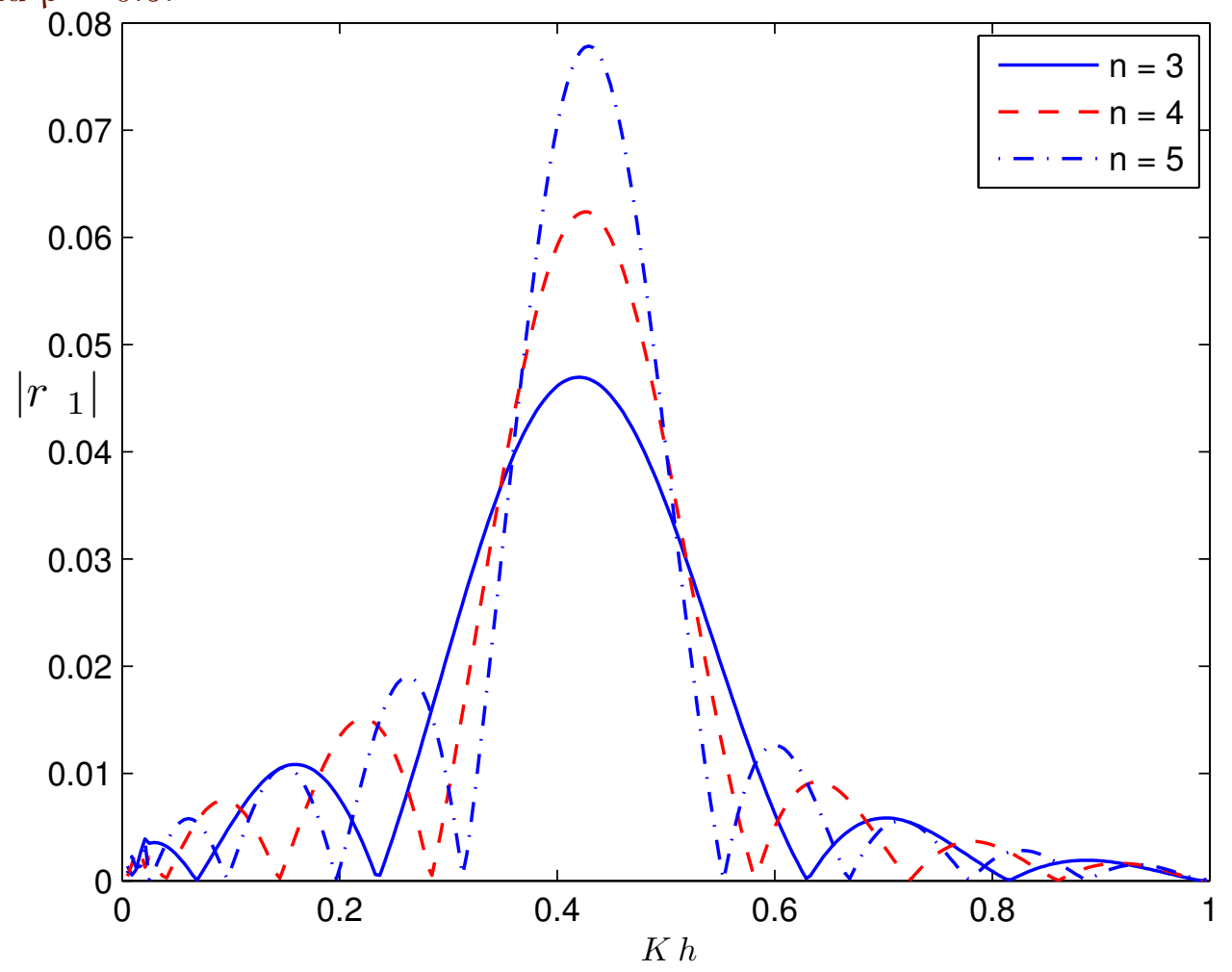

The different curves in Figures 5-7 correspond to different number of ripples on the bottom surface, when the surface tension parameter is fixed at $S / h^{2}=$ 0.001 and the density ratio is taken as 0.5 , for all these curves. From these figures, the peak values of reflection and transmission coefficients for the waves with wavenumber $p_{1}$ and $p_{2}$, due to a train of normal incident waves of wavenumber $p_{1}$ increase when the number of ripples increases. But when the number of ripples, $\mathrm{n}$, becomes large, the reflection and transmission coefficients become unbounded for certain values of $\mathrm{Kh}$. This is known as Bragg resonance which occurs when the reflection coefficient becomes much 
Figure 6: Reflection coefficient $\left|R_{1}\right|$ due to wave of mode $p_{1}$ for $S / h^{2}=0.001$ and $\rho=0.5$.

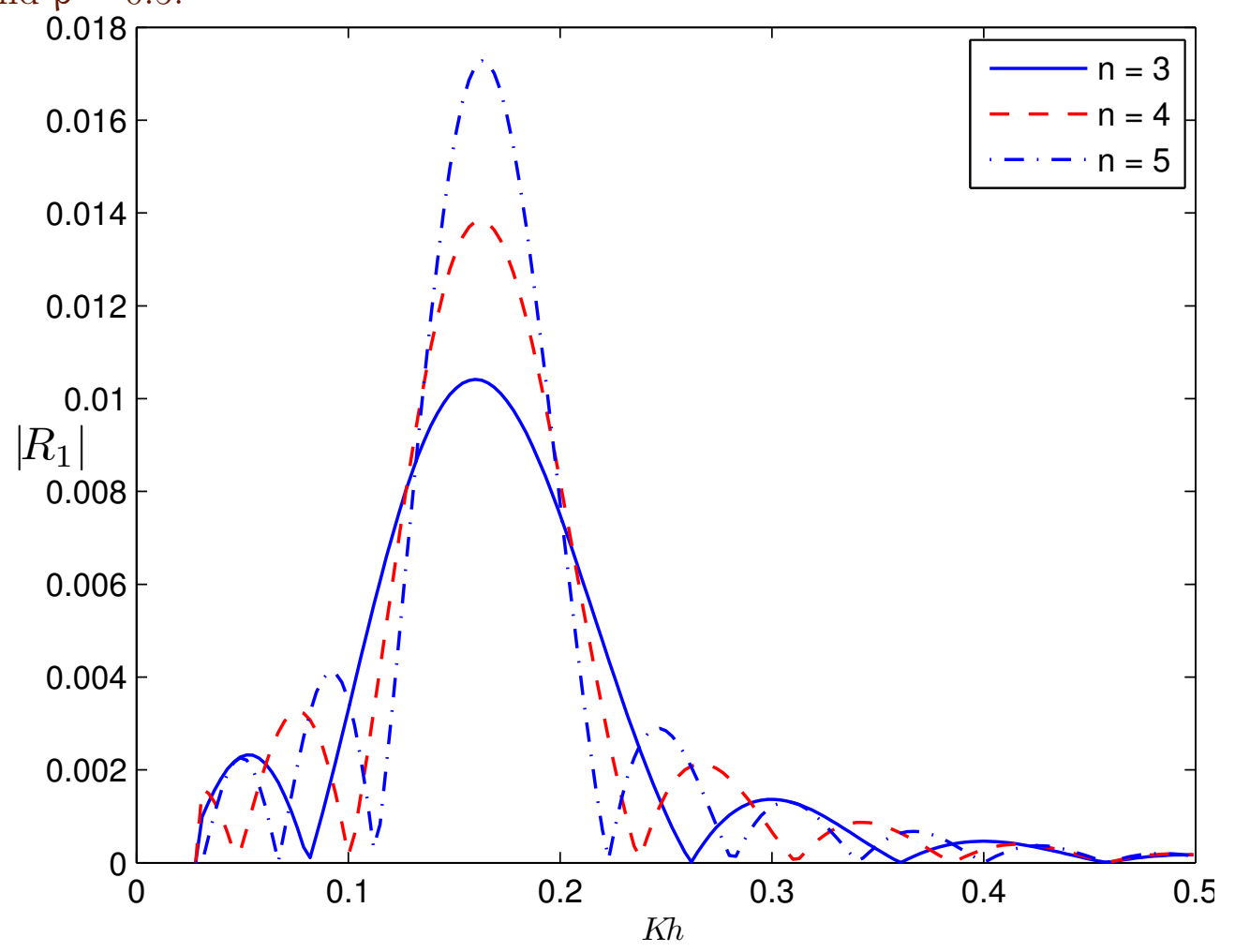

larger than the small parameter $\epsilon$. That means the perturbation expansion, which is discussed in section 3.1, ceases to be valid when the reflection coefficient becomes much larger than the undulation parameter, as pointed out by Mei [13].

Figures 8-10 show the different curves correspond to different density ratios, $\rho=0.5,0.6,0.9$, in a two-layer fluid flow region. In these figures $\mathrm{S} / \mathrm{h}^{2}=0.001$, $\mathrm{n}=3$ and $\mathrm{lh}=1$. Figure 8 shows that the peak values of the reflection coefficient are attained at different values of $\mathrm{Kh}$ (also the same observation can 
Figure 7: Transmission coefficient $\left|T_{1}\right|$ due to wave of mode $p_{1}$ for $S / h^{2}=$ 0.001 and $\rho=0.5$.

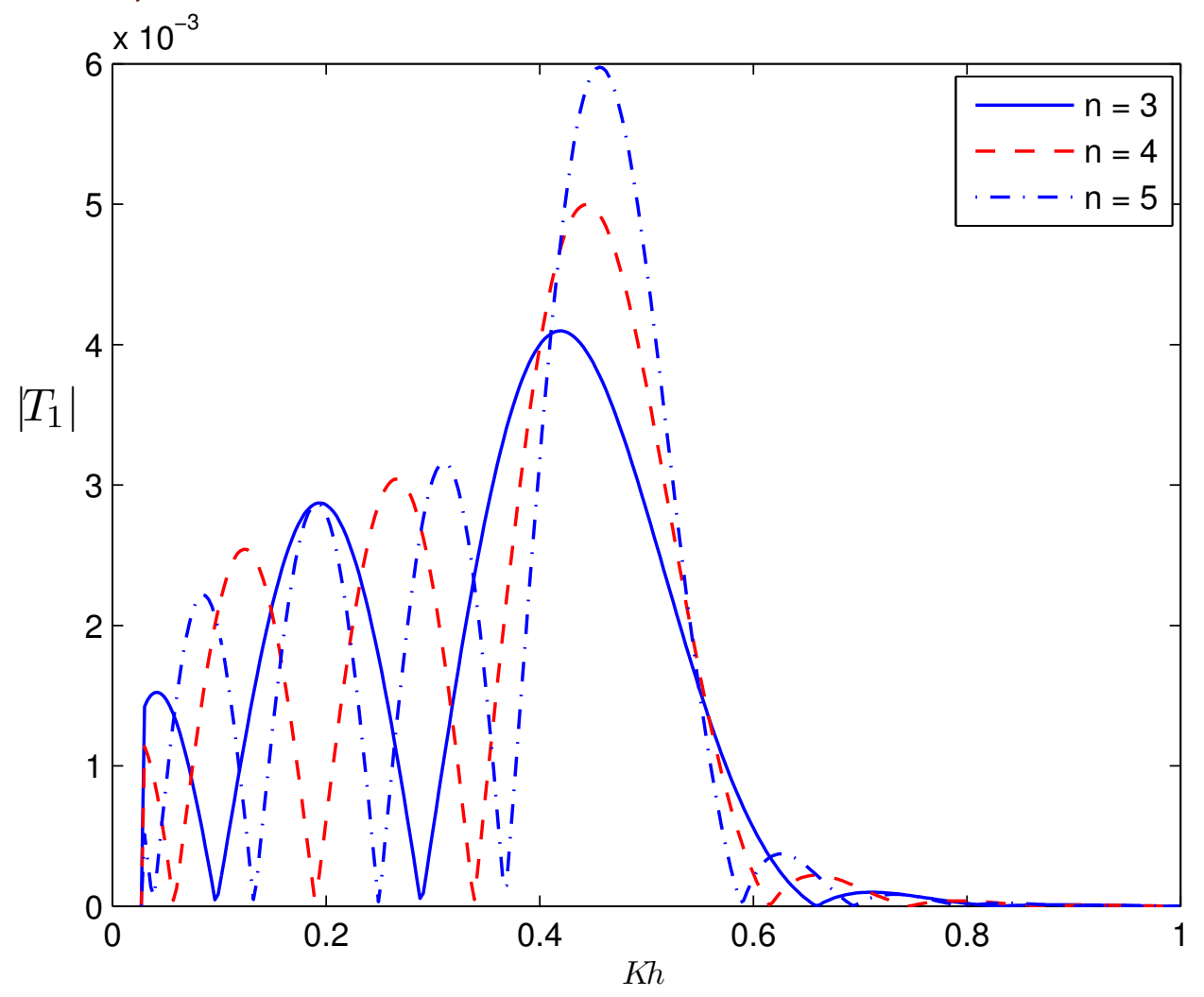


Figure 8: Reflection coefficient $\left|r_{1}\right|$ due to wave of mode $p_{1}$ for $S / h^{2}=0.001$ and $\eta_{0.05} 3$.

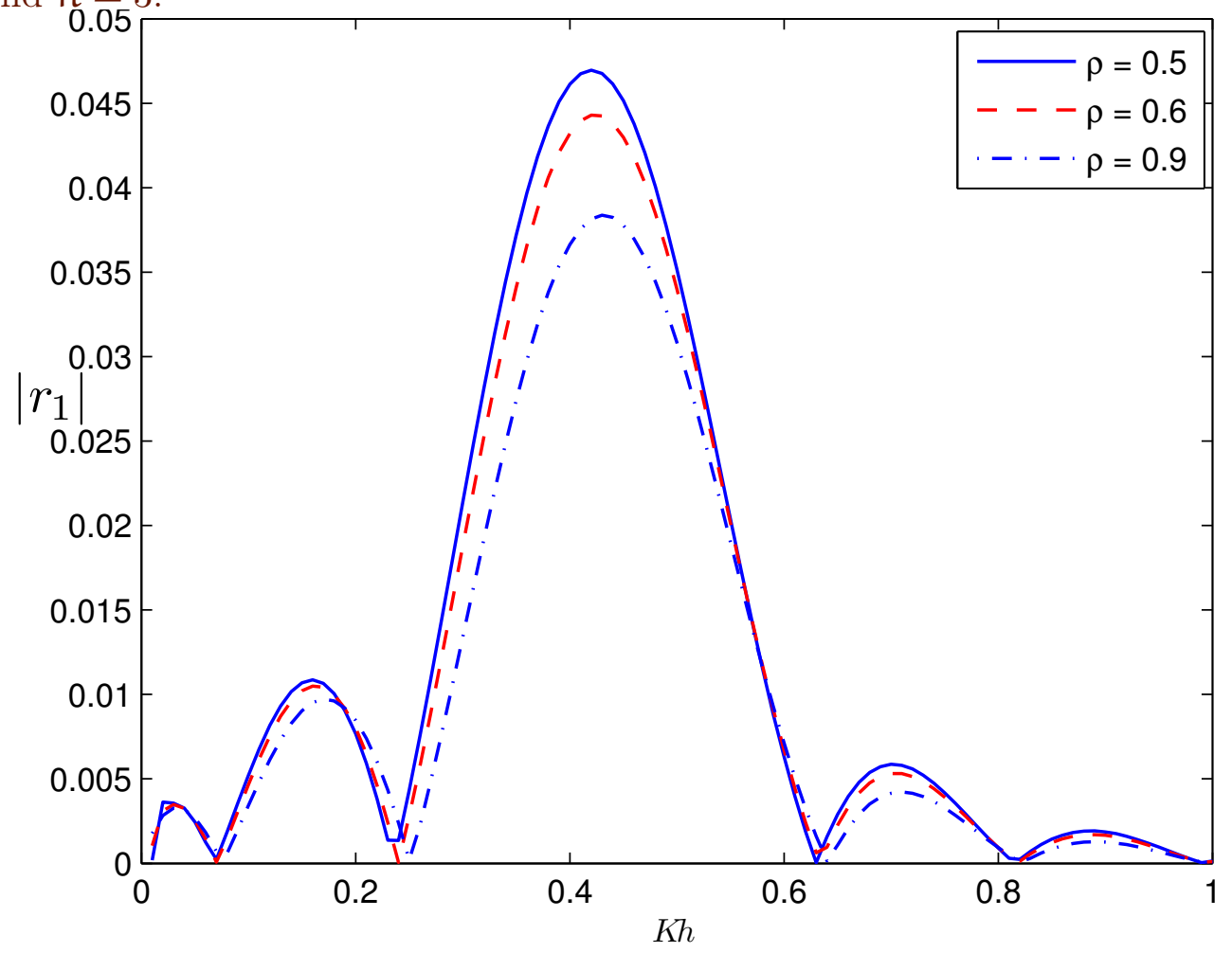

be made from the other curves in Figures 9 and 10). This happens due to the effect of density ratios in the dispersion relation which gives the wavenumbers of waves propagating in the positive $x$-direction in a two-layer fluid region. Since the wavenumbers become different for the change of density ratios, so the peak values of the reflection coefficient is attained at different values of $K h$. As the density ratio $\rho$ increases, the peak value of $\left|R_{1}\right|$ decreases, so that the first-order reflection coefficient is quite sensitive to the changes of density ratio due to normal incident waves with wave mode $p_{1}$ propagating in the positive $x$-direction on the bottom undulation. When the density ratio 
Figure 9: Reflection coefficient $\left|R_{1}\right|$ due to wave of mode $p_{1}$ for $S / h^{2}=0.001$ and $n=3$.

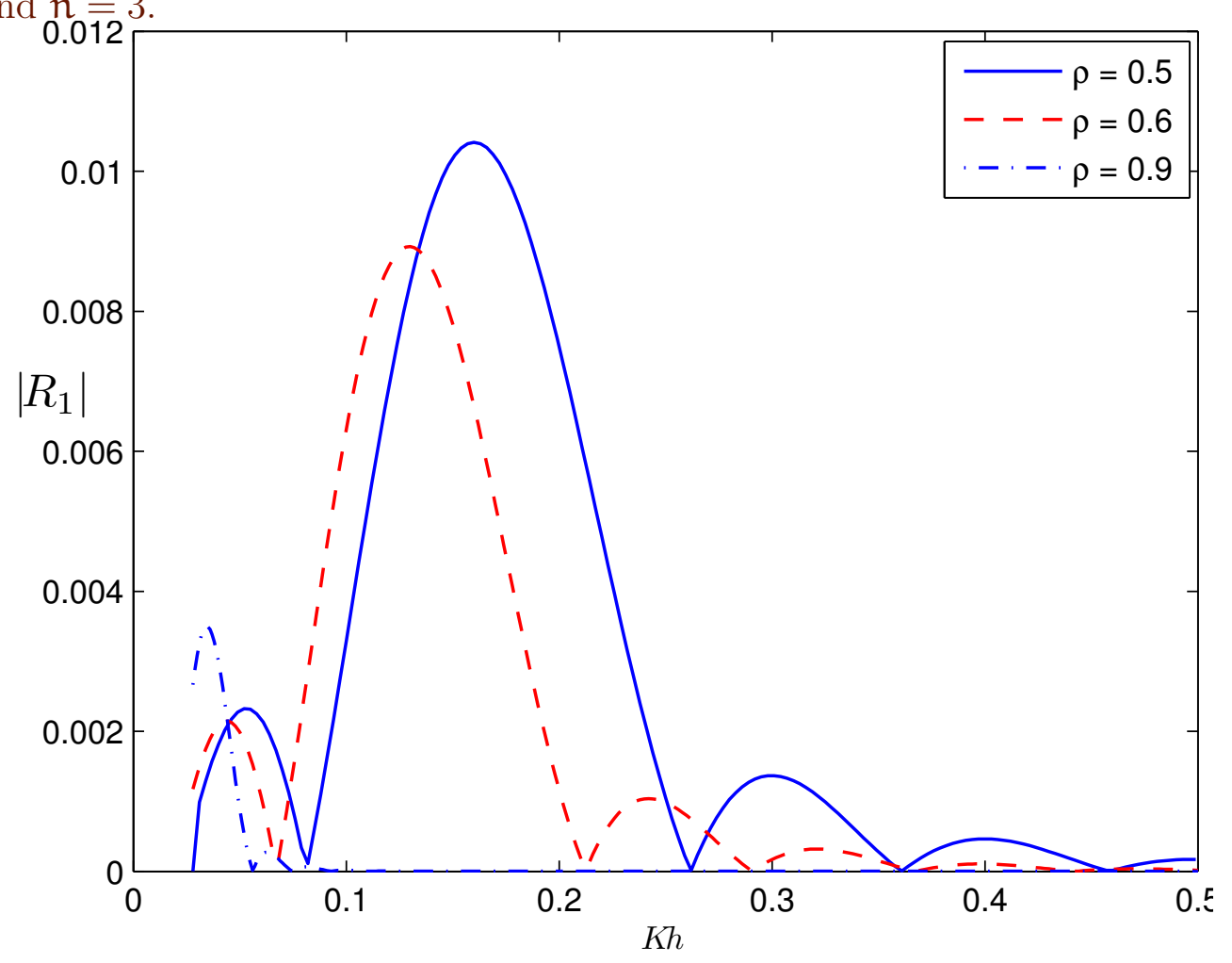

approaches the value one, the first-order reflection coefficient becomes smaller than those for the smaller density ratios.

From Figures 2-10, the number of zeros of reflection and transmission coefficients is much less in comparison to that of the example of Maiti and Mandal [11]. This happens due to the inclusion of the surface tension and the different ripple wave numbers in the patch of the undulation. Another common feature in these figures is the oscillating nature of the absolute values of the first-order coefficients as functions of $\mathrm{Kh}$. 
Figure 10: Transmission coefficient $\left|T_{1}\right|$ due to wave of mode $p_{1}$ for $S / h^{2}=$ 0.001 and $n=3$.

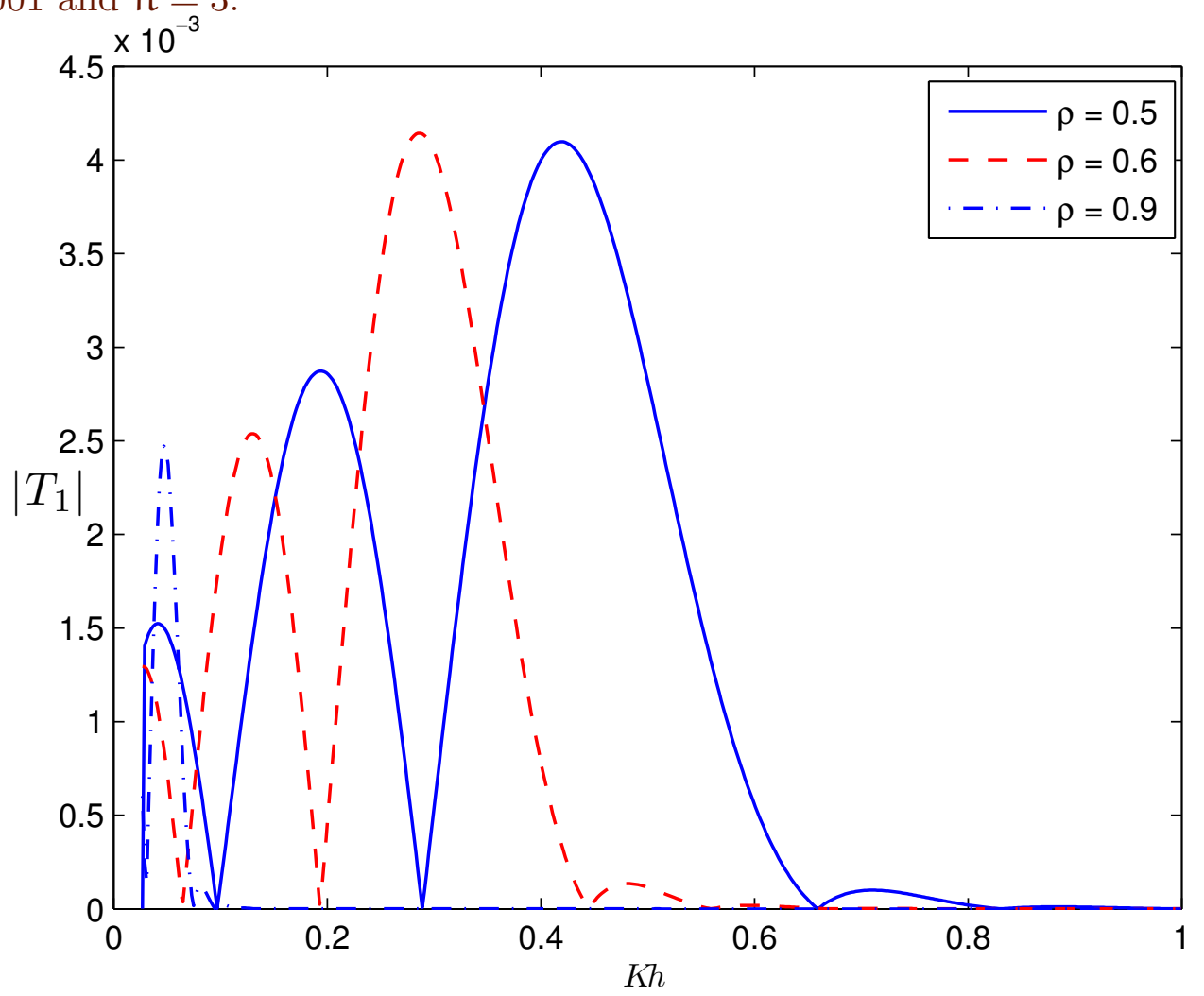

Similarly, the case of normal incident waves of wavenumber $p_{2}$ (an interfacial mode) is interesting due to the presence of surface tension where the energy is always possible to be converted from one wave mode to the other (like the normal incident wave of wavenumber $p_{1}$ ). For this case, Figures 11-19 show the first-order reflection coefficients $\left|r_{1}^{*}\right|,\left|R_{1}^{*}\right|$ and the transmission coefficient $\left|t_{1}^{*}\right|$ against $\mathrm{Kh}$ for $\mathrm{H} / \mathrm{h}=2, \mathrm{a} / \mathrm{h}=0.1$ and the ripple wavenumber $\mathrm{lh}=1$. The different curves in Figures 11 and 13, representing $\left|r_{1}^{*}\right|$ and $\left|t_{1}^{*}\right|$ of the waves of wavenumber $p_{1}$, for a train of normal incident waves with wavenumber $p_{2}$, 
Figure 11: Reflection coefficient $\left|r_{1}^{*}\right|$ due to wave of mode $p_{2}$ for $\rho=0.5$ and $\mathrm{n}=3$.

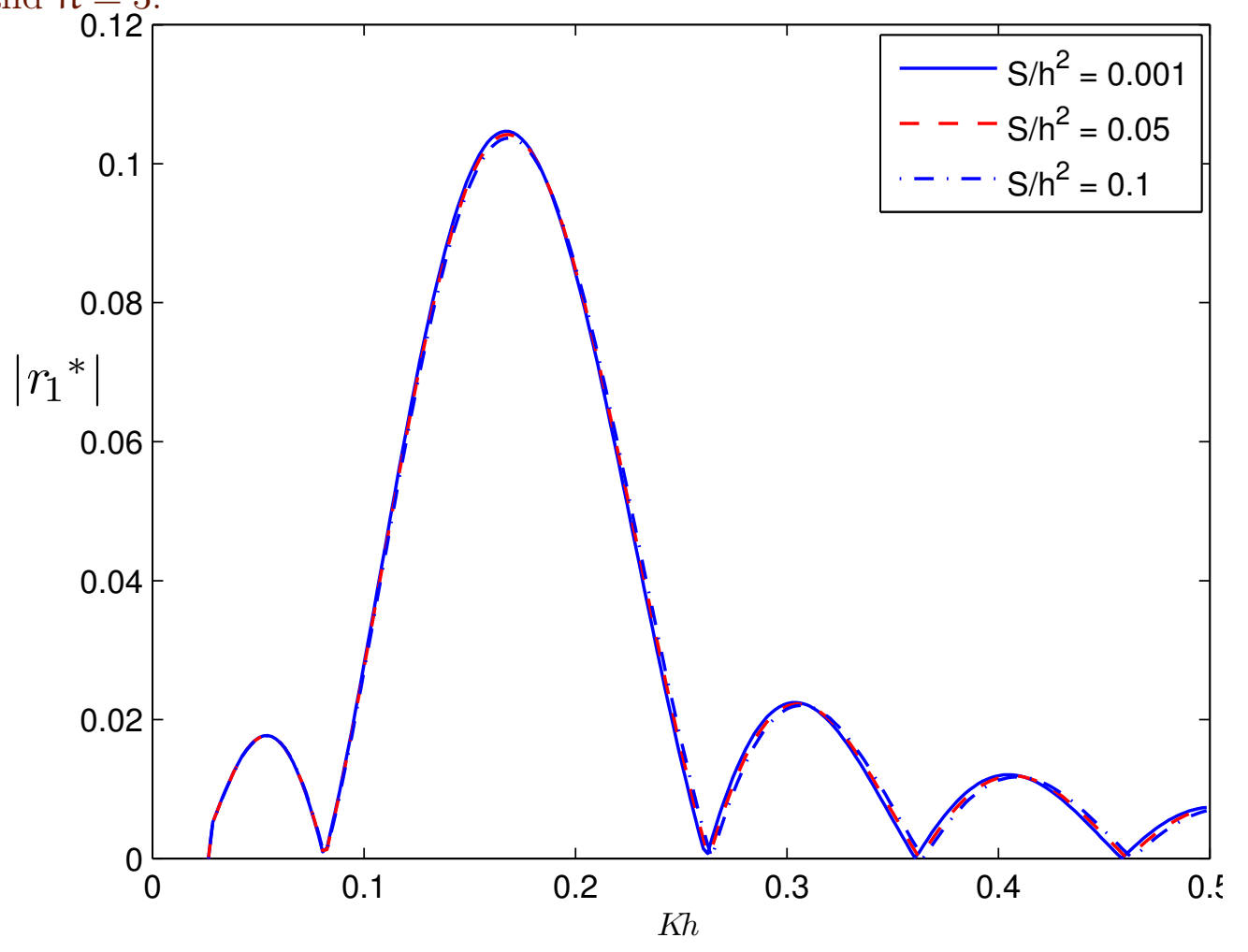

correspond to three different surface tension parameters $S / h^{2}=0.001,0.05$ and 0.1 , while $\mathrm{n}$ is fixed at 3 and the density ratio $\rho$ is 0.5 , for all these curves. These two figures show that as the surface tension parameter increases, the peak values of the reflection and transmission coefficients $\left|r_{1}^{*}\right|$ and $\left|t_{1}^{*}\right|$, respectively, decrease. Here, the decreasing rate of $\left|r_{1}^{*}\right|$ is negligible as compared to $\left|t_{1}^{*}\right|$. Figure 12 shows the reflection coefficient $\left|R_{1}^{*}\right|$ of the waves of wavenumber $\mathrm{p}_{2}$, for the normal incident wave of wavenumber $\mathrm{p}_{2}$, corresponding to different surface tension parameters. As the values of surface tension parameter $S / h^{2}$ increase, the peak values of reflection coefficient $\left|R_{1}^{*}\right|$ also in- 
Figure 12: Reflection coefficient $\left|R_{1}^{*}\right|$ due to wave of mode $p_{2}$ for $\rho=0.5$ and $n=3$.

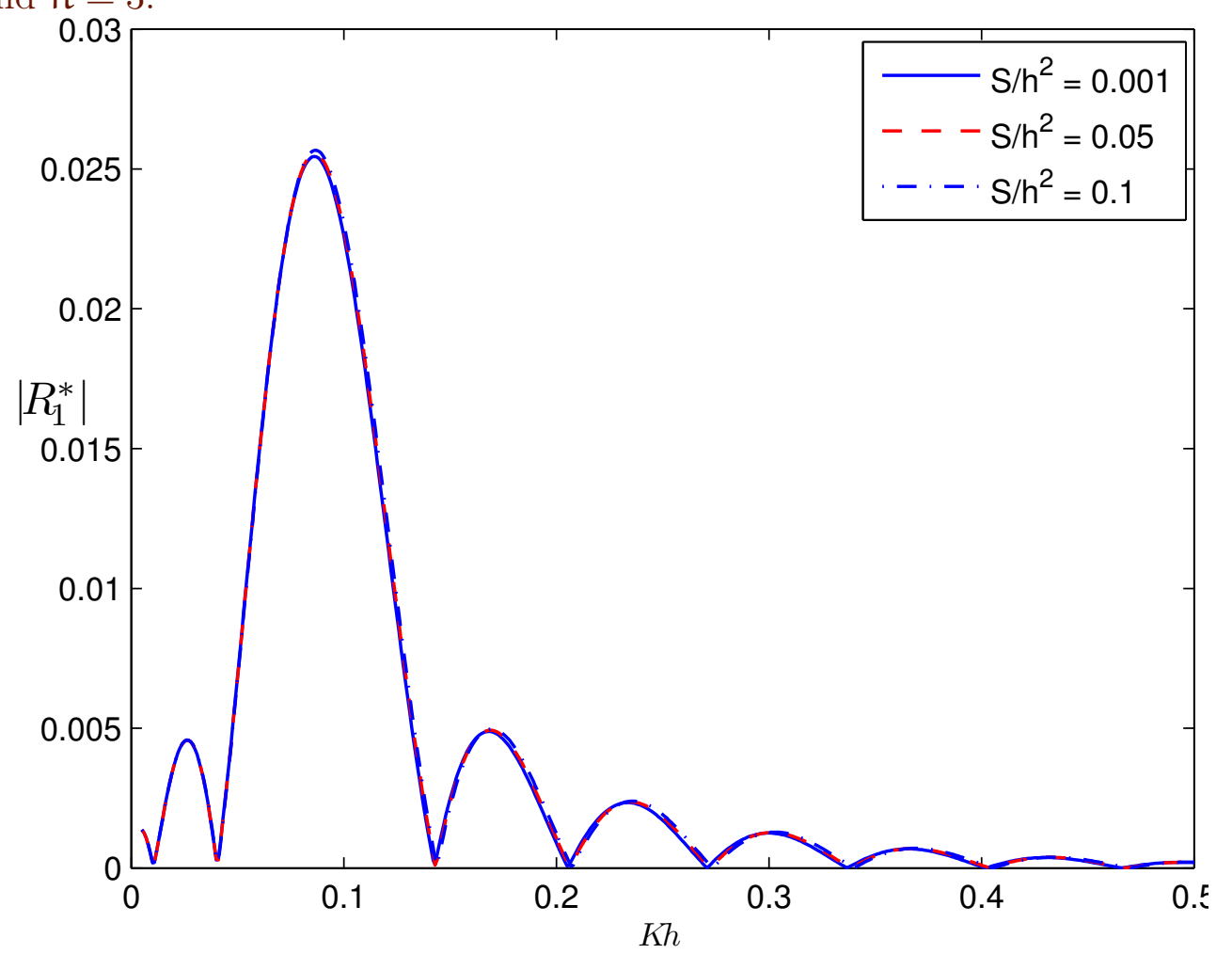

crease. Also the non-zero values of the reflection and transmission coefficients (from Figures 11-13) due to the normal incident waves of wavenumber $p_{2}$ show that there is always a possibility of some conversion of energy from one wavenumber to the other.

The different curves in Figures 14-16 showing the reflection and transmission coefficients corresponding to different number of ripples on the bottom surface while the surface tension parameter is fixed at $S / h^{2}=0.001$ and the density ratio is fixed at 0.5. These figures show that as the number of ripples increases, the reflection coefficient $\left|r_{1}^{*}\right|$ (for waves with wavenumber $p_{1}$ ), $\left|R_{1}^{*}\right|$ 
Figure 13: Transmission coefficient $\left|t_{1}^{*}\right|$ due to wave of mode $p_{2}$ for $\rho=0.5$ and $n=3$.

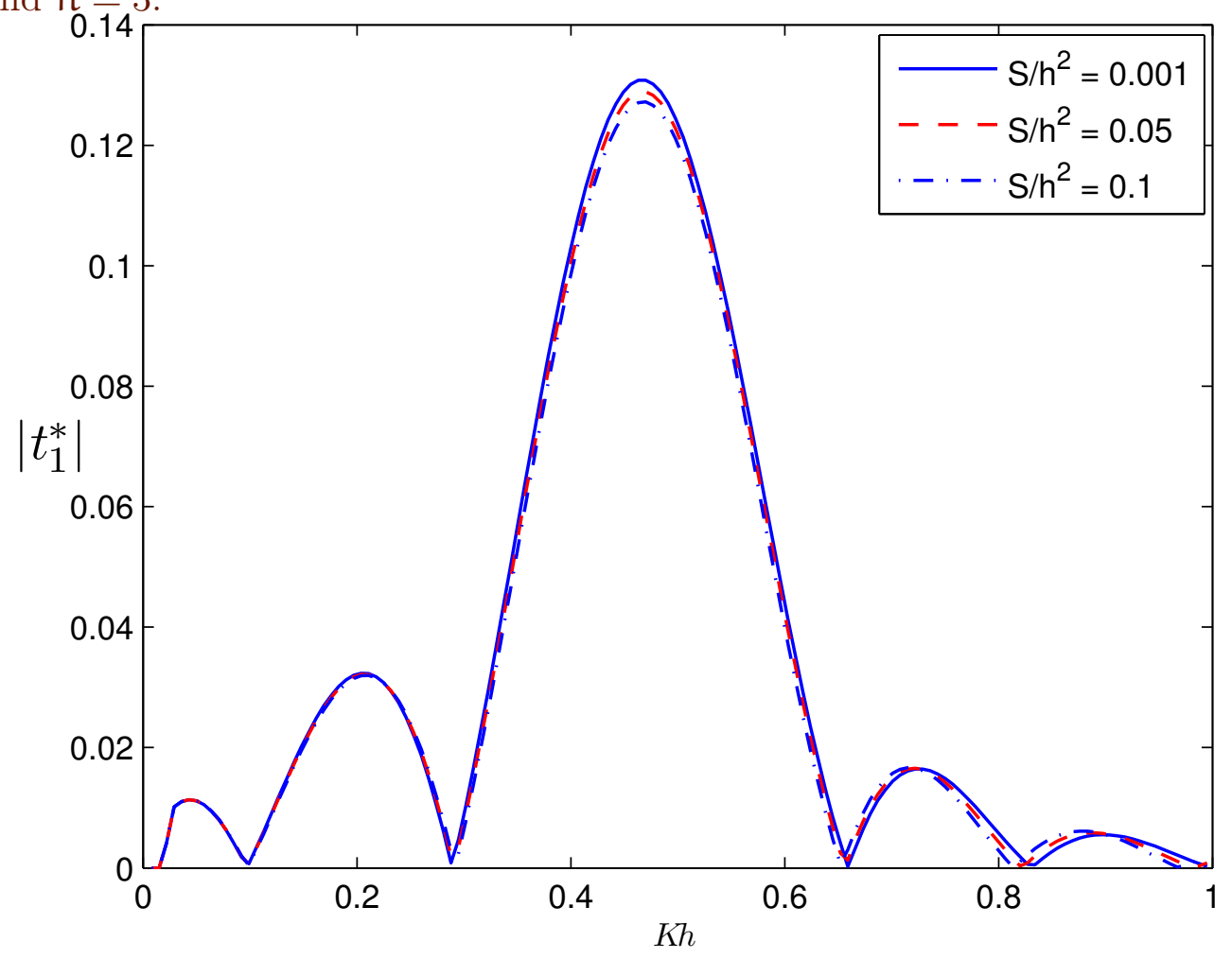

(for waves with wavenumber $p_{2}$ ) and transmission coefficient $t_{1}^{*}$ (for waves with wavenumber $p_{1}$ ) due to normal incident waves with wavenumber $p_{2}$ always exists and their peak values increase accordingly. But when the number of ripples $\mathrm{n}$ becomes large, the reflection and transmission coefficients become unbounded for certain values of $\mathrm{Kh}$. Therefore, in this mode $\mathrm{p}_{2}$ also, such a perturbation expansion, which is discussed in Section 3.1, ceases to be valid at Bragg resonance which occurs when the reflection coefficient becomes much larger than the undulation parameter. 
Figure 14: Reflection coefficient $\left|r_{1}^{*}\right|$ due to wave of mode $p_{2}$ for $S / h^{2}=0.001$ and $\rho=0.5$.

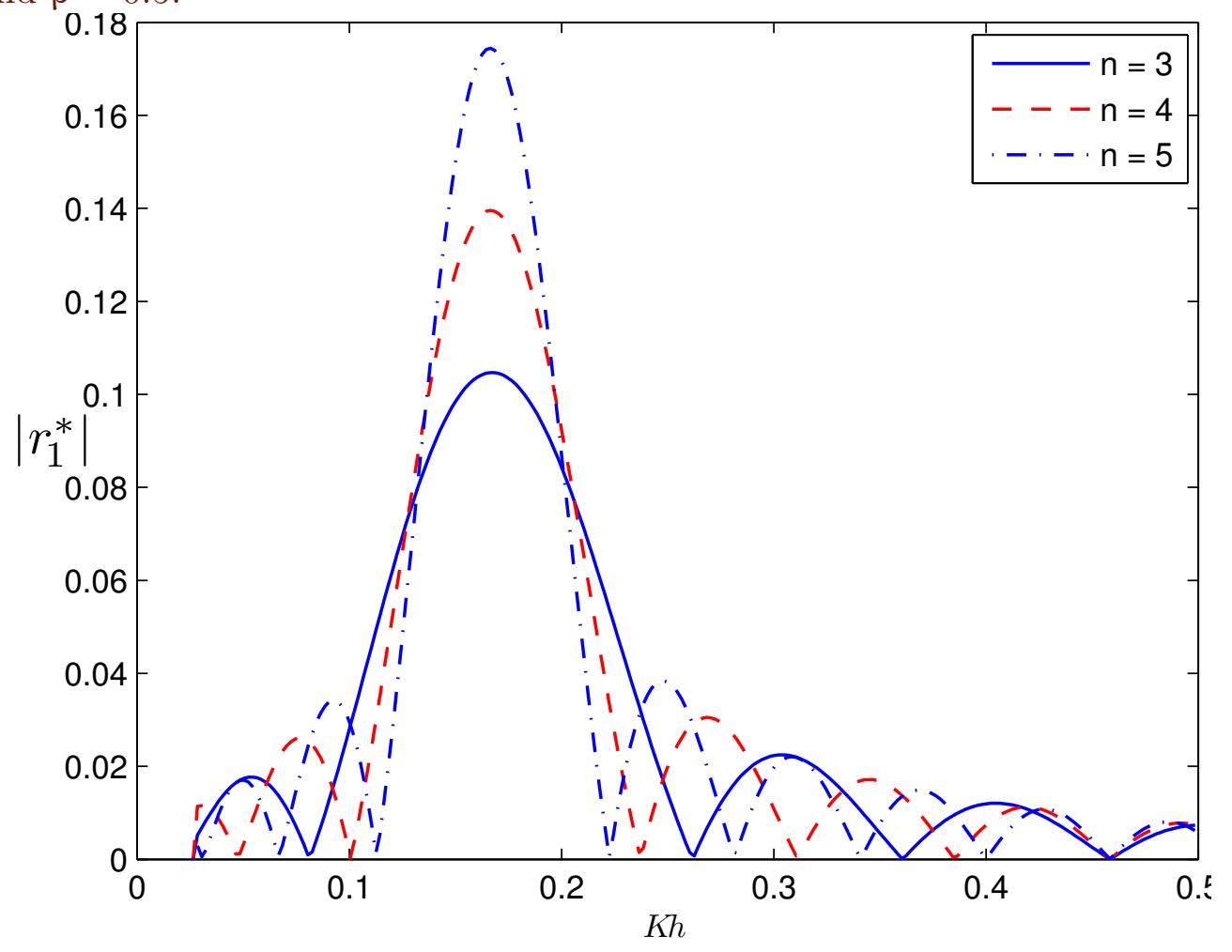

In Figures 17-19, different curves correspond to different density ratios $\rho=0.5$, 0.6 and 0.9 in a two-layer fluid flow region. In these figures, $\mathrm{S} / \mathrm{h}^{2}=0.001$ and $n=3$. Figure 17 shows that the peak values of the reflection coefficient are attained at different values of $\mathrm{Kh}$ (also the same observation can be made from the other curves in Figures 18 and 19). The reason for this happening is same as in the case of normal incident waves of wavenumber $p_{1}$. Also, we observe from Figure 17 that as the density ratio $\rho$ increases, the peak values of reflection and transmission coefficients $\left|r_{1}^{*}\right|,\left|R_{1}^{*}\right|$ and $\left|t_{1}^{*}\right|$ increase. That means the first-order reflection and transmission coefficients are quite sensitive to 
Figure 15: Reflection coefficient $\left|R_{1}^{*}\right|$ due to wave of mode $p_{2}$ for $S / h^{2}=0.001$ and $\rho=0.5$.

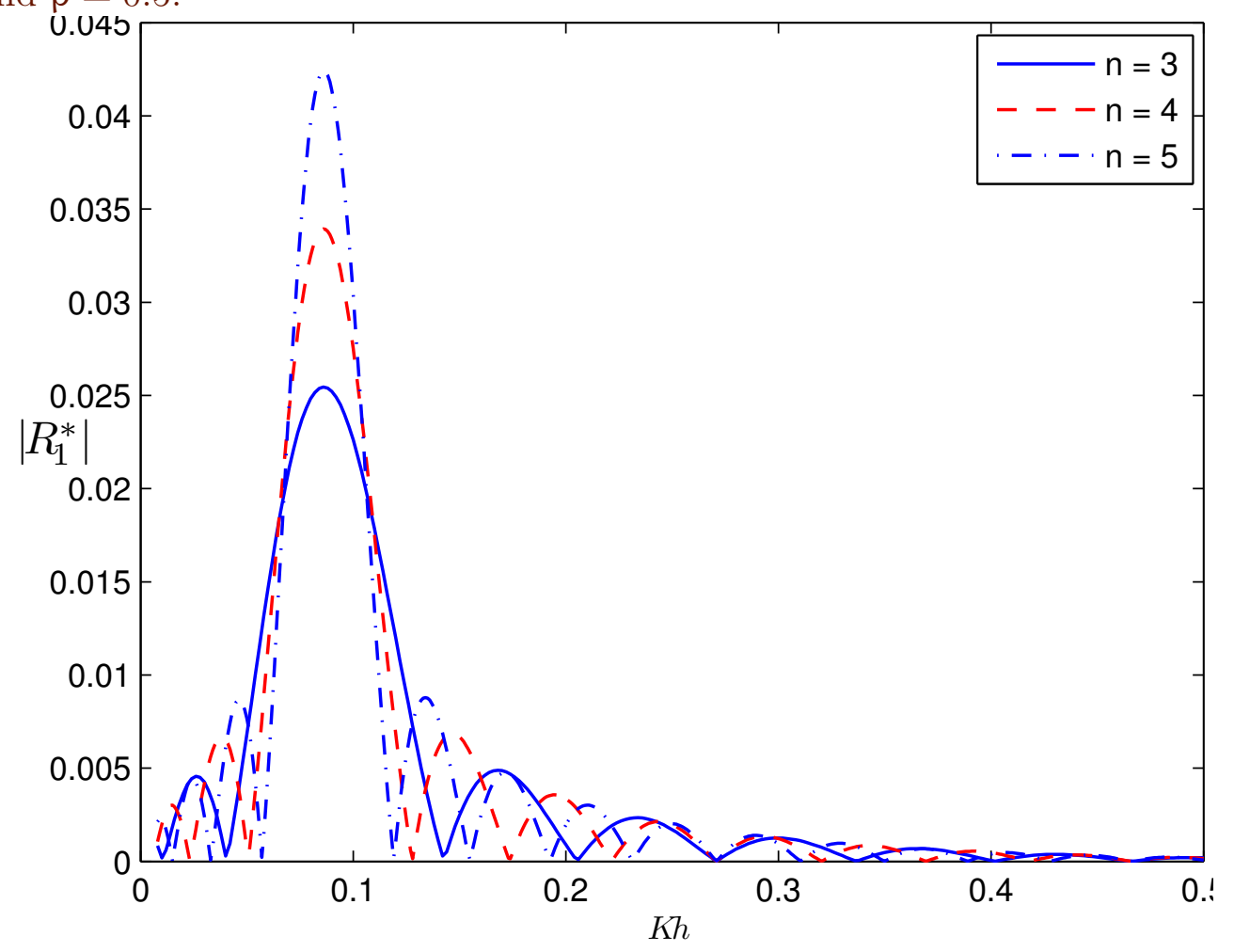

the change of density ratios due to normal incident waves of wavenumber $p_{2}$ propagating in the positive $x$-direction on the bottom undulation. When the density ratio approaches the value one, the first-order reflected and transmitted energy become more than those for the smaller density ratios. At the same time, the number of zeros of reflection and transmission coefficients become more. Moreover, the non-zero values of reflection and transmission coefficients in Figures 11-19 show that some conversion of energy from one wavenumber to the other is always possible. 
Figure 16: Transmission coefficient $\left|t_{1}^{*}\right|$ due to wave of mode $\mathrm{p}_{2}$ for $\mathbf{S} / \mathrm{h}^{2}=$ 0.001 and $\rho=0.5$.

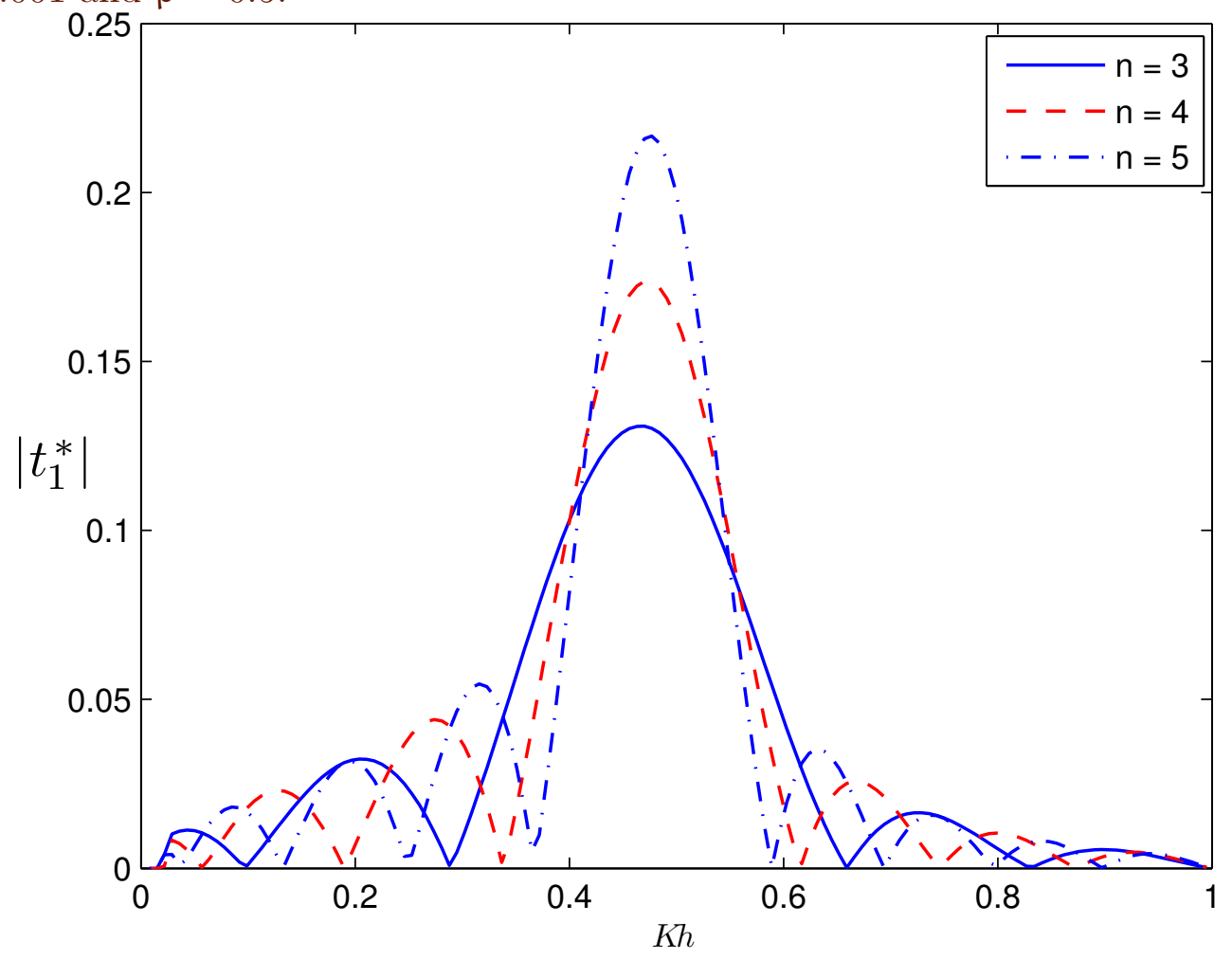

For all the results obtained throughout our work, it is reasonable to compare them with the work of Maiti and Mandal [11], as these two problems bear similarities to a reasonable extent. There is one basic difference in the problem formulation of the present work and the work of Maiti and Mandal [11]. While the work of Maiti and Mandal [11] there was a free-surface, our work presents a problem where the presence of surface tension on the free-surface has been taken into account. Therefore, it is to be expected that there must be some common features among these two works. 
Figure 17: Reflection coefficient $\left|r_{1}^{*}\right|$ due to wave of mode $p_{2}$ for $S / h^{2}=0.001$ and $\mathrm{n}=3$.

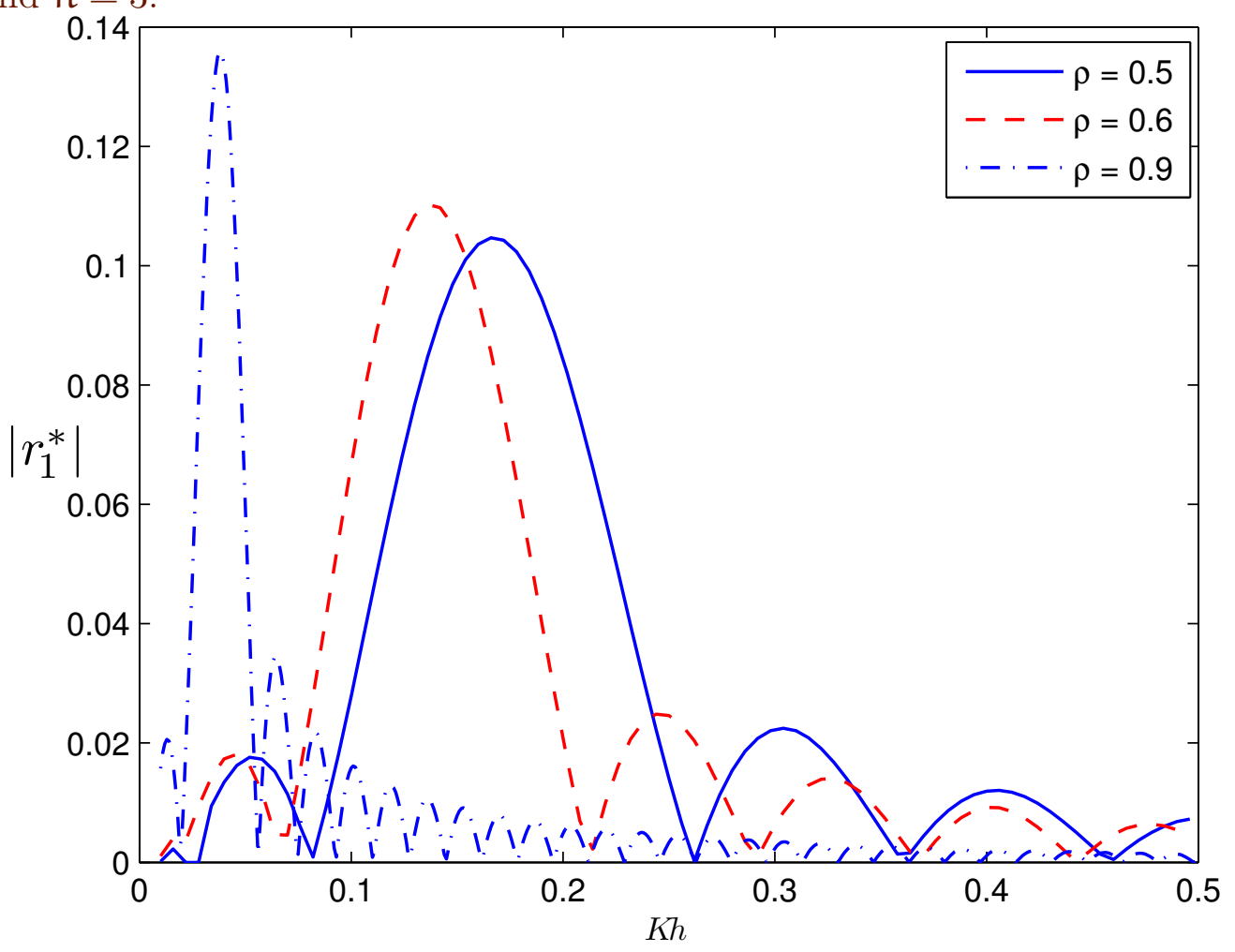

\section{Conclusion}

In the present study, the scattering of normal incident waves by a small bottom undulation of a two-layer fluid in the presence of surface tension at the free-surface is investigated by employing a simplified perturbation analysis. The determination of the first-order correction to the velocity potentials and, hence, the reflection and transmission coefficients become easier while employing the Green's integral theorem with the introduction of appropriate Green's functions. For the particular case of a patch of sinusoidal 
Figure 18: Reflection coefficient $\left|R_{1}^{*}\right|$ due to wave of mode $p_{2}$ for $S / h^{2}=0.001$ and $n=3$.

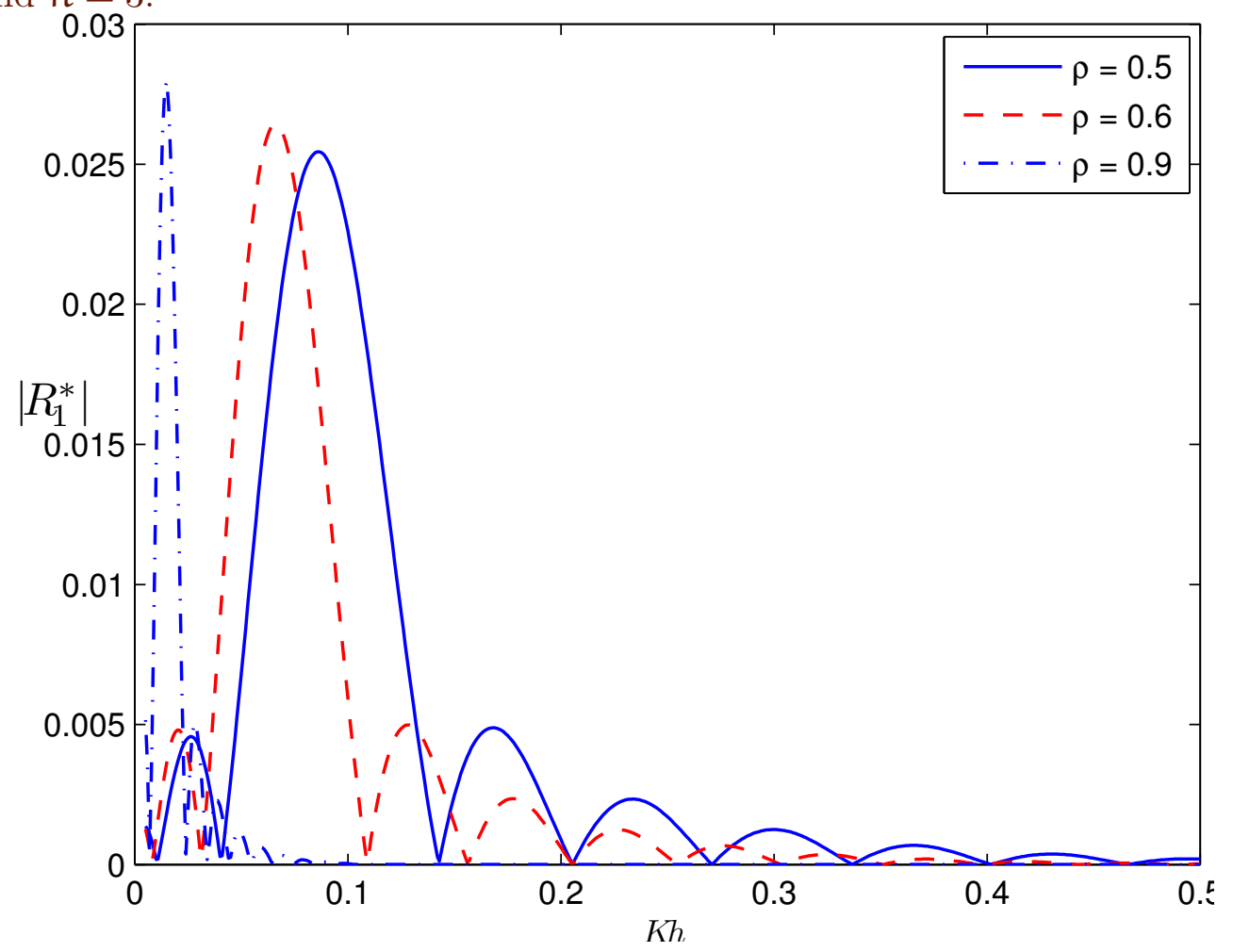

ripples at the bottom surface, the first-order correction to the reflection and transmission coefficients are depicted in a number of figures. When a train of incident waves is of free-surface wave mode, then the reflection corresponding to the free-surface wave mode decreases while the reflection and transmission corresponding to interfacial wave mode increase due to increase of surface tension at the free-surface. But when a train of incident waves is an interfacial wave mode, then the reflection and transmission corresponding to free-surface wave mode decrease, whereas the reflection corresponding to interfacial wave mode increases due to increase of surface tension parameters. 
Figure 19: Transmission coefficient $\left|t_{1}^{*}\right|$ due to wave of mode $p_{2}$ for $\mathbf{S} / \mathbf{h}^{2}=$ 0.001 and $\mathrm{n}=3$.

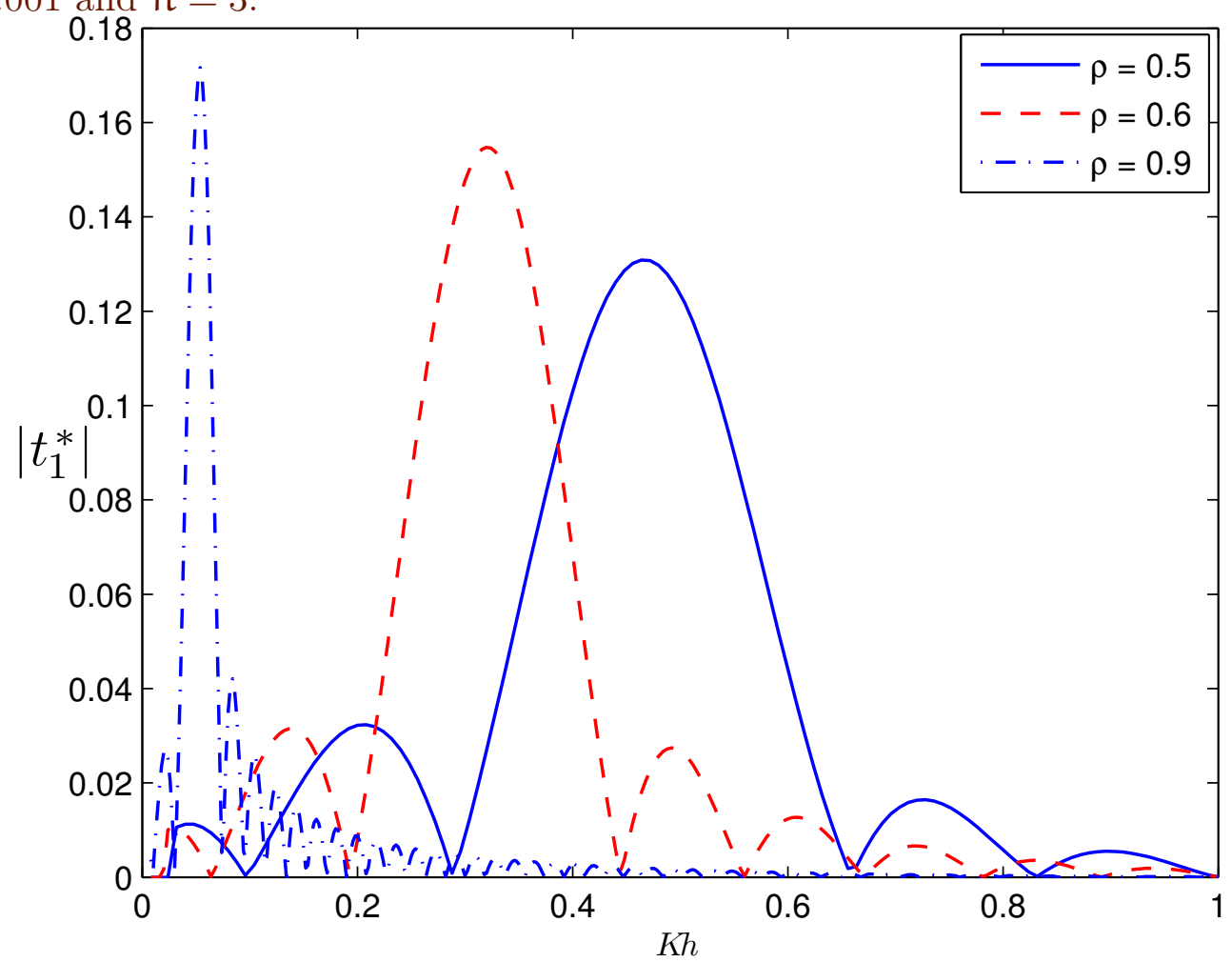

Again, the peak values of reflection and transmission coefficients for both the modes increase with the number of ripples of the sinusoidal bottom undulation. When a train of incident waves is of a free-surface wave mode, then the reflection corresponding to both the modes decreases whereas the transmission corresponding to interfacial wave mode increases due to increase of density ratios in the two-layer fluid. But when a train of incident waves is of interfacial wave mode, then the reflection and transmission for both the modes increase due to increase of density ratios. The results obtained here are expected to be qualitatively helpful in tackling two-layer fluid problems in 
the presence of surface tension at the free-surface in an ocean with an uneven bottom surface.

Acknowledgement The author thanks Prof. Swaroop Nandan Bora, Department of Mathematics, Indian Institute of Technology Guwahati, India, for his valuable discussions and suggestions to carry out the preparation of the manuscript. The author expresses his deep gratitude to the referees for their invaluable comments and suggestions which enabled the author in carrying out the desired revision of the manuscript. This work is partially supported by a research grant from Department of Science and Technology, India (No. SB/FTP/MS-003/2013).

\section{References}

[1] P. G. Chamberlain and D. Porter, "Wave scattering in a two-layer fluid of varying depth", J. Fluid Mech. 524 (2005) 207-228; doi:10.1017/S0022112004002356 E41

[2] G. J. D. Chapman and R. Porter, "Second-order sloshing over an arbitrary bed", J. Fluid Mech. 524 (2005) 331-355; doi:10.1017/S0022112004002502 E41, E48

[3] A. G. Davies, "The reflection of wave energy by undulations of the sea bed", Dyn. Atmos. Oceans 6 (1982) 207-232; doi:10.1016/0377-0265(82)90029-X E41, E57

[4] D. V. Evans, "The influence of surface tension on the reflection of water waves by a plane vertical barrier", Proc. Camb. Phil. Soc. 64 (1968) 795-810; doi:10.1017/S0305004100043504 E41

[5] D. V. Evans, "The effect of surface tension on the waves produced by a heaving circular cylinder", Proc. Camb. Phil. Soc. 64 (1968) 833-847; doi:10.1017/S030500410004353X E41, E44 
[6] R. Harter, I. D. Abrahams and M. J. Simon, "The effect of surface tension on trapped modes in water wave problems" Proc. R. Soc. Lond. A 463, (2007) 147-180; doi:10.1098/rspa.2007.0063 E41

[7] R. Harter, M. J. Simon and I. D. Abrahams, "The effect of surface tension on localized free-surface oscillations about surface-piercing bodies", Proc. R. Soc. A 464 (2008) 3039-3054; doi:10.1098/rspa.2008.0059 E41

[8] H. Lamb, Hydrodynamics, (6th ed. Cambridge University Press, Cambridge, 1932). E41

[9] C. M. Linton and M. McIver, "The interaction of waves with horizontal cylinders in two-layer fluids", J. Fluid Mech. 304 (1995) 213-229; doi:10.1017/S002211209500440X E41, E59

[10] C. M. Linton and J. R. Cadby, "Scattering of oblique waves in a two-layer fluid", J. Fluid Mech. 461 (2002) 343-364; doi:10.1017/S002211200200842X E42, E45

[11] P. Maiti and B. N. Mandal, "Scattering of oblique waves by bottom undulations in a two-layer fluid", J. Appl. Math. \& Computing 22 (2006) 21-39; doi:10.1007/BF02832035 E41, E45, E46, E53, E56, E60, E67, E74

[12] B. N. Mandal and S. De, Water wave scattering (CRC Press, Florida, 2015). E41

[13] C. C. Mei, "Resonant reflection of surface water waves by periodic sandbars", J. Fluid Mech. 152 (1985) 315-335; doi:10.1017/S0022112085000714 E41, E48, E49, E58, E64

[14] J. W. Miles, "Oblique surface wave diffraction by a cylindrical obstacle", Dyn. Atmos. Oceans 6 (1981) 121-123; doi:10.1016/0377-0265(81)90019-1 E41 
[15] S. Mohapatra and S. N. Bora, "Water wave interaction with a sphere in a two-layer fluid flowing through a channel of finite depth", Arch. Appl. Mech. 79 (2009) 725-740; doi:10.1007/s00419-008-0248-z E41

[16] Mohapatra, S. and Bora, S. N. Scattering of internal waves in a two-layer fluid flowing through a channel with small undulations. Ocean Dynamics, 59 (2009) 615-625; doi:10.1007/s10236-009-0214-5 E41, E45

[17] S. Mohapatra and S. N. Bora, "Reflection and transmission of water waves in a two-layer fluid flowing through a channel with undulating bed", Z. Angew. Math. Mech.[ZAMM] 91(1) (2011) 46-56; doi:10.1002/zamm.200800216 E41, E45

[18] S. Mohapatra and S. N. Bora, "Propagation of oblique waves over small bottom undulation in an ice-covered two-layer fluid", Geophysical and Astrophysical Fluid Dynamics 103 (2009) 347-374; doi:10.1080/03091920903071077 E41, E46, E50, E56

[19] R. Porter and D. Porter, "Scattered and free waves over periodic beds", J. Fluid Mech. 483 (2003) 129-163; doi:10.1017/S0022112003004208 E41

[20] P. F. Rhodes-Robinson, "On the forced surface waves due to a vertical wave-maker in the presence of surface tension" Math. Proc. Camb. Phil. Soc. 70 (1971) 323-337; doi:10.1017/S0305004100049926 E41, E42

[21] P. F. Rhodes-Robinson, "Note on the reflexion of water waves at a wall in the presence of surface tension", Math. Proc. Camb. Phil. Soc. 92 (1982) 369-374; doi:10.1017/S0305004100060011 E41

\section{Author address}

1. Smrutiranjan Mohapatra, Department of Mathematics, Veer Surendra Sai University of Technology, Burla-768 018, India mailto:smrutiranjan.m@gmail.com 